\title{
Contamination in plastic recycling: Influence of metals on the quality of reprocessed plastic
}

Kampmann Eriksen, Marie; Pivnenko, Kostyantyn; Olsson, Mikael Emil; Fruergaard Astrup, Thomas

Published in:

Waste Management

Link to article, DOI:

10.1016/j.wasman.2018.08.007

Publication date:

2018

Document Version

Peer reviewed version

Link back to DTU Orbit

Citation (APA):

Kampmann Eriksen, M., Pivnenko, K., Olsson, M. E., \& Fruergaard Astrup, T. (2018). Contamination in plastic recycling: Influence of metals on the quality of reprocessed plastic. Waste Management, 79, 595-606.

https://doi.org/10.1016/j.wasman.2018.08.007

\section{General rights}

Copyright and moral rights for the publications made accessible in the public portal are retained by the authors and/or other copyright owners and it is a condition of accessing publications that users recognise and abide by the legal requirements associated with these rights.

- Users may download and print one copy of any publication from the public portal for the purpose of private study or research.

- You may not further distribute the material or use it for any profit-making activity or commercial gain

- You may freely distribute the URL identifying the publication in the public portal 
3

4

\author{
M. K. Eriksen", K. Pivnenko, M. E. Olsson, T. F Astrup \\ Department of Environmental Engineering, Technical University of Denmark, DK-2800 \\ Kgs. Lyngby, Denmark
}

*) Corresponding author: Marie Kampmann Eriksen

Department of Environmental Engineering, Technical University of Denmark, DK-2800 Kgs.

Lyngby, Denmark

maker@env.dtu.dk

Declarations of interest: none 


\section{Abstract}

The global consumption of plastic continues to increase, and plastic recycling is highlighted as crucial to saving fossil resources and closing material loops. Plastic can be made from different polymers and contains a variety of substances, added intentionally to enhance the plastic's properties (metals added as fillers, colourants, etc.). Moreover, plastic can be contaminated during use and subsequent waste management. Consequently, if substances and contaminants are not removed during recycling, potentially problematic substances might be recycled with the targeted polymers, hence the need for quantitative data about the nature and presence of these substances in plastic. Samples of selected polymers (PET, PE, PP, PS) were collected from different origins; plastic household waste, flakes/pellets of reprocessed plastic from households and industry, and virgin plastic. Fifteen selected metals (Al, $\mathrm{As}, \mathrm{Cd}, \mathrm{Co}, \mathrm{Cr}, \mathrm{Cu}, \mathrm{Fe}$, $\mathrm{Hg}, \mathrm{Li}, \mathrm{Mn}, \mathrm{Ni}, \mathrm{Pb}, \mathrm{Sb}, \mathrm{Ti}, \mathrm{Zn}$ ) were quantified and the statistical analysis showed that both the polymer and origin influenced the metal concentration. $\mathrm{Sb}$ and $\mathrm{Zn}$ were potentially related to the production stage of PET and PS, respectively. Household plastic samples (waste and reprocessed) were found to contain significantly higher $\mathrm{Al}, \mathrm{Pb}$, Ti and $\mathrm{Zn}$ concentrations when compared to virgin samples. Only the concentration of Mn was reduced during washing, suggesting that parts of it was present as physical contamination. While most of the metals were below legal limit values, elevated concentrations in reprocessed plastic from households, aligned with increasing recycling rates, may lead to higher metal concentrations in the future.

Keywords: Circular economy, household waste (HHW), compositional data, post-consumer plastic, toxic metals

Abbreviations $^{1}$

\footnotetext{
${ }^{1}$ FOD: Frequency of detection, HDPE: High density polyethylene, HHW: Household waste, IW: Industrial waste, LDPE: Low density polyethylene, LOD: Limit of detection, MANOVA: Multivariate analysis of variance, PE: Polyethylene, PET: Polyethylene terephthalate, PP: Polypropylene, PS: Polystyrene
} 


\section{Introduction}

Continuously increasing resource consumption in modern societies, where resources are often lost after the first life cycle, places pressure on the finite resources of the globe. As a measure to partially mitigate this, and to ensure more sustainable development, the concept of a circular economy, where resources are recirculated into society after being used, has been adopted by many legal authorities (e.g. EC, 2015; Government of Japan, 2010). The European Union (EU) has presented a strategy towards a circular economy that has a strong focus on material flows and subsequent recycling, to keep materials in the loop (EC, 2015). In the strategy, plastic has been highlighted as a priority area, since it is an important material for modern societies, is used in large quantities and is predominantly made from fossil fuels. Consequently, the strategy dictates increases in European recycling rates for both plastic packaging and plastic from household waste (HHW). In addition, the EU has developed a circular economy strategy solely for plastic, further stressing the importance of product design and high-quality recycling (EC, 2018a).

Plastic waste is a complex and heterogeneous material stream, due to several factors. First, plastic as material refers to numerous different polymers with different chemical properties that need to be separated from each other prior to recycling. The main polymers found in plastic from HHW are polyethylene terephthalate (PET), polyethylene (PE), polypropylene (PP) and polystyrene (PS) (Edjabou et al., 2015), collectively representing $63.2 \%$ of the plastic demand in Europe (PlasticsEurope and EPRO, 2017). Second, many different additives can be introduced during the production phase to control the properties of the plastic and make it fulfil the requirements for use in specific applications. These include additives such as colourants, fillers, plasticisers, lubricants, antioxidants (Hahladakis et al., 2018), commonly used in plastic packaging (Lahimer et al., 2013), as well as additives such as flame retardants, commonly used in plastic for electronics (Hansen, 2013). While plastic 
packaging represents a significant share of plastic in HHW (Edjabou et al., 2015), waste electronics end up in HHW primarily due to miss-sorting (Edjabou et al., 2015). The type and content of these additives is regulated to varying degrees when it comes to use in specific applications. As an example, plastic used in food packaging needs to comply with the most strict and comprehensive legislation with respect to chemical composition and migration of potentially problematic substances (EU, 2011b). Consequently, plastic applicable for food contact is in this study defined as "high-quality". Thereby, this term refers to the potential applicability (and "circularity" of the plastic) with respect to legal requirements for chemical composition, rather than the physical and mechanical properties of the material (melt flow index, impact strength, etc.). In contrast to high-quality plastic, "low-quality" is used to characterize plastic applicable only for applications with less strict requirements in relation to chemical composition or migration (electrical and electronic equipment, non-food packaging, etc.). Hence, plastic in such applications might contain higher concentrations of potentially problematic substances. Consequently, the chemical properties and the quality of the plastic can vary depending on the specific product and its application. Third, substances can be added non-intentionally, either in the production phase (e.g. residues from catalysts, metal impurities in non-metal additives (Lahimer et al., 2017)) or as contamination through potential sorption during use and waste management (Pivnenko and Astrup, 2016). Some contaminants might be chemically embedded in the plastic matrix rather than being present as physical contamination ("dirt") that can be removed during recycling, e.g. during washing of the plastic waste. Consequently, there is a risk of recycling not only the desired plastic material, but also potentially problematic substances, ultimately affecting the applicability and quality of the reprocessed plastic material. This phenomenon has been demonstrated quantitatively for other materials (e.g. paper (Pivnenko et al., 2016a)), and several sources have underlined the 
importance of a "clean" circular economy in relation to plastic recycling (Ellen MacArthur Foundation, 2016; Goldberg, 2017).

In regards to available literature on plastic from households, the most analysed items are PET water bottles approved for food contact, with both the total content and migration of potentially problematic substances being studied. The findings are summarised in a review by Bach et al. (2012) which reveals that several substances have been shown to migrate from PET bottles into bottled water, such as $\mathrm{Sb}$, acetaldehylde and selected phthalates, despite the fact that e.g. phthalates are not added intentionally during PET plastic production. However, the migration never exceeded either European or American limit values. PET bottles for mineral water or carbonated drinks represent a relatively homogeneous material stream in which all the items are similar in shape and design and comply with strict legislation related to food packaging. Streams of mixed plastic waste, however, are much more heterogeneous and contaminated (Ragaert et al., 2017), and not all items have to meet the aforementioned strict legislation. Nonetheless, very few studies have chemically analysed HHW streams including plastic items other than bottles, or plastic recovered from HHW and reprocessed into pellets or flakes, representing recycled raw plastic material. Camacho and Karlsson (2000) analysed samples of high-density PE (HDPE) and PP waste sorted from mixed HHW for low-molecular weight organic substances. They identified the presence of several substances related to cosmetics, cleaning agents and personal hygiene in the HDPE waste that was not present in virgin HDPE (including selected acids, esters and alcohols). Moreover, Pivnenko et al. (2016b) found that the concentration of selected phthalates was higher in reprocessed plastic and plastic from HHW, compared to virgin plastic and reprocessed plastic from industrial waste (IW). Similarly, Huber and Franz (1997) found high concentrations of phthalates in reprocessed HDPE made from HHW bottles. All the literature sources mentioned above suggest that plastic 
115 from HHW might contain contaminants with the potential to impair the quality of recycled plastic material.

Most studies focused on the presence or migration of organic substances as contaminants. While organic substances may degrade or migrate during use and recycling, inorganic substances, such as metals, are in most cases expected to persist in the material after recycling (Hansen, 2013), though small amounts might migrate during use (Whitt et al., 2016; Bach et al., 2012). Several metals are currently intentionally added during plastic production (often as oxides, acids, etc. (Hahladakis et al., 2018; Hansen, 2013)). These include additives such as colourants (containing $\mathrm{Ti}, \mathrm{Cr}, \mathrm{Co}, \mathrm{Cd}, \mathrm{Pb}, \mathrm{Zn}, \mathrm{Fe}, \mathrm{Al}, \mathrm{Cu}$ ), antioxidants and stabilisers (containing $\mathrm{Cd}, \mathrm{Pb}, \mathrm{Zn}$ ) or other additives (containing $\mathrm{As}, \mathrm{Li}, \mathrm{Pb}, \mathrm{Cd}, \mathrm{Zn}, \mathrm{Sb}, \mathrm{Al}$ ) (Hahladakis et al., 2018; Hansen, 2013). Moreover, metals in plastic can originate from catalysts used in plastic production (e.g. $\mathrm{Sb}, \mathrm{Ti}, \mathrm{Cr}, \mathrm{Hg}, \mathrm{Mn}$ ), or contamination added or sorbed to the plastic during production, use and waste management (e.g. Fe, $\mathrm{Al}, \mathrm{Cu}, \mathrm{Mn}, \mathrm{Zn}, \mathrm{Ni}$ ) (Hahladakis et al., 2018; Hansen, 2013; Bach et al., 2012; Romão et al., 2010; EC, 2008). As most of these metals have well-documented toxic effects and/or can be classified as persistent and bioaccumulative (Goldberg, 2017; EC, 2008), it is desirable to reduce recycling of metals in plastic, in order to minimise potential health risks and deterioration of material quality. Where the form of the metals, their hazardousness and exposure influence the potential risk to human health and the environment, the total metal content can be used to identify potential deterioration of material quality, which affects the applicability of the recycled plastic and thereby the circularity (Eriksen et al., 2018). Currently very limited knowledge exists about the fate of metals during plastic recycling, and metal contamination in plastic has been assessed previously only by focusing on one single source of plastic, polymer or metal (Götze et al., 2016; Bach et al., 2012; Romão et al., 2010). Consequently, there is a need to quantify and document the total metal content in conjunction with the plastic material quality, rather than potential health risks, as a 
140 first step towards coherently assessing the metal content in plastic collected from various steps in the recycling chain.

The aim of this work was to provide consistent quantitative data on the presence of selected metals in plastic samples of different origins and polymer types with the purpose of identifying potential deterioration of material quality and applicability. The waste plastic samples were further divided into food and non-food contact items, as the substances added to the plastic are expected to be different for the two product groups due to stricter legislation for food contact items. Finally, the impact of pre-treatment through washing was assessed on the waste samples. The differences in metal concentrations between the sample groups were analysed statistically and the effects of metal concentration on the applicability and quality of the reprocessed plastic were evaluated. This was achieved through the following specific objectives: 1) obtain samples of relevant polymer types (PET, PE, PP and PS) from different origins; plastic waste from households (HHW), reprocessed plastic waste (pellets or flakes) from households and industry as well as virgin plastic; 2) pre-treat waste plastic samples for analysis, including separation into food and non-food contact items, sample shredding and washing; 3) analyse the concentration of selected metals (Al, $\mathrm{As}, \mathrm{Cd}, \mathrm{Co}, \mathrm{Cr}, \mathrm{Cu}, \mathrm{Fe}, \mathrm{Hg}, \mathrm{Li}$, $\mathrm{Mn}, \mathrm{Ni}, \mathrm{Pb}, \mathrm{Sb}, \mathrm{Ti}, \mathrm{Zn}$ ) in all samples; 4) assess statistical differences in metal concentrations between the different sample groups; and 5) evaluate levels of metal contamination and their potential influence on the applicability of reprocessed waste plastic in material recycling.

\section{Materials and methods}

\subsection{Sample overview}

Eighty-two samples of plastic waste, reprocessed plastic and virgin plastic were collected from a range of sources. Table 1 provides an overview of the collected plastic samples, assigned sample group, sample origin, polymer type, etc. The samples were of the polymer types PET, 
PE, PP and PS and belonged to one of four main sample groups, which differed regarding their origin.

Figure 1 illustrates where in the plastic life cycle the samples in the different sample groups were obtained as well as where intentional addition and contamination of metals can occur. While contamination can occur during all steps of the recycling chain, intended addition takes place only during the production of raw material and plastic products. Samples in sample groups 1a and $1 \mathrm{~b}$ were derived from waste plastic streams, whereas samples in groups 2-4 were collected as pellets or flakes, representing raw material for plastic product production. Consequently, samples in group 1 represented raw material from which reprocessed plastic pellets or flakes can be produced, thus before the actual reprocessing step. Samples in groups 2-4 represented raw material from which plastic products can be produced, hence after (re)processing but before conversion into products. Since no data was available regarding the past and future applications of samples in groups 2-4, addressing application as a factor for metal content was not possible in the present work. However, as more additives are often added during conversion of raw material into products, to reach the desired properties for that specific product, the authors find that comparison between samples of virgin plastic (group 4) and reprocessed plastic (group 2 and 3) is valid. This is based on the assumption that the future application will only have minor influence on the metal content in these samples, as they were collected before conversion into products. 
While waste plastic samples (group 1) were pre-treated before being analysed, samples of reprocessed and virgin plastic (group 2-4) were analysed as received. Further details about sample collection and pre-treatment are provided in Section 2.1.1.

\subsubsection{Collection and pre-treatment}

Samples of source-separated HHW plastic (group 1a) were collected from two Danish sorting facilities, one pilot scale facility sorting source-separated plastic from the Municipality of Copenhagen (Nederskov, 2017) and one full-scale facility sorting source-separated, commingled recyclables (plastic and metal) from households in Northern Jutland (Winther, 2017). The two facilities sorted the rigid plastic waste mechanically into the polymer types PET, PE (primarily HDPE), PP, PS, soft plastic and mixed residues (including plastic). Streams of soft plastic and mixed residual plastic represented a mixture of polymers with an unknown composition (although primarily low density PE (LDPE) for soft plastic). As post-consumer waste plastic streams with unknown composition are especially challenging to recycle (Ragaert et al., 2017), samples of soft and mixed residual plastic were excluded from this study. Subsamples of each polymer type (between 2 and $28 \mathrm{~kg}$ ) were collected systematically over a single day and sorted manually into items representing food and non-food contact items, which were identified from the label on the product or the cup/fork symbol that guarantees that the item has been approved for food contact items (EC, 2004). As an example, a ketchup bottle was sorted as food contact items, whereas a shampoo bottle was sorted as non-food contact items. Items without a label or cup/fork symbol were sorted as non-food applications. This resulted in sample subgroups of food and non-food contact items for each polymer with sample sizes of 0.25 to $20 \mathrm{~kg}$.

To homogenise the material, samples were shredded coarsely, followed by 1D splitting to reduce the sample size. 1D splitting is a mass reduction technique that aims to ensure 
representativeness of the final sample and reduce errors in sampling when compared to other mass reduction techniques (Dahlén and Lagerkvist, 2008; Lagerkvist et al., 2011). Additionally, it has been used previously for a variety of waste materials, including garden and household waste (Boldrin and Christensen, 2010; Götze et al., 2016). In this study, it was performed on a flat pile of coarsely shredded plastic that was split into equally sized increments, using a plastic box and a shovel. Every second increment was discarded, reducing the sample size by half until a sample size of approximately $1 \mathrm{~kg}$ was achieved. 1D splitting was only performed on samples with an initial size $>1.5 \mathrm{~kg}$. Finally, the reduced samples were finely shredded into flakes $<10 \mathrm{~mm}$.

The surface of plastic waste might be physically contaminated with metal particles from the use, collection and waste management phases. As most recycling processes are expected to include a washing step (Ragaert et al., 2017; Luijsterburg and Goossens, 2014), physical contamination may be washed off and thereby removed during waste plastic reprocessing. Previous experiments with waste plastic washing suggested metal removal efficiencies between $5 \%$ and $77 \%$ (Cossu et al., 2012). Hence, a washing step was performed on half of the samples from group $1 \mathrm{a}$ and $1 \mathrm{~b}$, to identify metals partially present as physical contaminants rather than being chemically embedded in the plastic. During the washing experiment, finely shredded plastic samples $(0.5 \mathrm{~kg})$ were put in contact with $5 \mathrm{~L}$ of demineralised (Milli-Q) water on a turning table at room temperature. After 24 hours of washing, the water phase was decanted and plastic samples dried at $50^{\circ} \mathrm{C}$ for 24 hours, before being analysed.

Samples from group 1b were originated from Danish residual mixed HHW, where no sorting system was in place. From an initial sample of $930 \mathrm{~kg}$, the plastic waste was sorted manually, based on polymer identification marks (PET, PE, PP and PS) on the plastic products. Due to the large sample size, each polymer type was filled in randomly numbered paper sacks (140 L), and every paper sack with an odd number was discarded. Following the simple random 
sampling approach (US EPA, 2002), the procedure was repeated until a desired sample size of

$240 \quad 5-10 \mathrm{~kg}$ for each of the polymer types. The remaining steps included coarse shredding, 1D

241 splitting, fine shredding and washing, following the same approach as described for sample

242 group 1a. All the sorting and pre-treatment steps for sample groups 1a and 1b are summarised

243 in Figure 2.

Groups 2 and 3 contained reprocessed plastic waste from households and industry, respectively. Plastic HHW entails post-consumer plastic that has been through a use phase in a household, whereas plastic from IW entails either pre-consumer waste from the industry (e.g. off cuts) or waste from the industry that has been through a use phase (e.g. plastic wrapping around bales of straw, used in the agricultural sector). The samples were collected from several plastic recycling companies from Europe and China, and most of them were in the form of pellets, and therefore assumed to be mixed and homogenised; hence, they were analysed without any pre-treatment. As previously mentioned, more detailed knowledge related to the origin of the plastic waste, as well as intended applications, was not available. Group 4 contained virgin plastic samples collected from large plastic-producing companies in Europe and China in form of pellets or flakes, and so they did not require pretreatment prior to chemical analysis. The virgin samples were included in the analysis as reference materials to which the concentrations in the reprocessed and waste plastic samples were compared.

\subsection{Chemical analysis}


All plastic samples were analysed for the total content of the following 15 selected metals: Al, As, $\mathrm{Cd}, \mathrm{Co}, \mathrm{Cr}, \mathrm{Cu}, \mathrm{Fe}, \mathrm{Hg}, \mathrm{Li}, \mathrm{Mn}, \mathrm{Ni}, \mathrm{Pb}, \mathrm{Sb}, \mathrm{Ti}$ and $\mathrm{Zn}$. These metals were chosen, as they may be present in plastic, due to one or more of the following reasons: 1) intentionally added

266

267 as part of an additive (Al, $\mathrm{As}, \mathrm{Cd}, \mathrm{Co}, \mathrm{Cr}, \mathrm{Cu}, \mathrm{Fe}, \mathrm{Li}, \mathrm{Pb}, \mathrm{Sb}, \mathrm{Ti}, \mathrm{Zn}$ ), 2) used as catalysts in production ( $\mathrm{Sb}, \mathrm{Ti}, \mathrm{Cr}, \mathrm{Hg}, \mathrm{Mn}$ ) or 3) added from contamination during use and waste management (Fe, Al, Cu, Mn, Zn, Ni) (Hahladakis et al., 2018; Hansen, 2013; Bach et al., 2012; Romão et al., 2010; EC, 2008). Additionally, at least half of these metals (As, Cd, Co, Cr, Hg, $\mathrm{Pb}, \mathrm{Sb}, \mathrm{Ti}$ ) are known to be used in plastic in substances classified as carcinogenic, mutagenic, toxic for reproduction and/or dangerous for the environment (EC, 2008; ECHA, 2017; Hahladakis et al., 2018; Hansen, 2013). A detailed list of the specific substances in which the selected metals are used and their hazard classification is provided in Appendix A, Supplementary Material (SM).

\subsubsection{Sample preparation and digestion}

For each sample, triplicates containing $0.1 \mathrm{~g}(+/-0.02 \mathrm{~g})$ were prepared, adding up to 243 samples in total. All samples were digested by microwave-assisted digestion (Multiwave 3000, Anton-Paar), using 3 mL H2 $\mathrm{SO}_{4}$ (99.999\% Sigma-Aldrich) and $6 \mathrm{~mL} \mathrm{HNO}_{3}$ (65\% Suprapure ${ }^{\mathrm{TM}}$ grade, Merck). The power was set to $600 \mathrm{~W}$, with a full rotor of 16 vessels and a pressure increase at $0.3 \mathrm{bar} / \mathrm{second}$ over a $15-\mathrm{min}$ ramp, with the maximum pressure set to 24 bar and the maximum temperature $210^{\circ} \mathrm{C}$. The power was held for 90 min and magnetic stirring was applied to all samples during digestion. Subsequently, the samples were diluted to $100 \mathrm{~mL}$ using MillQ grade water. To keep $\mathrm{Hg}$ in suspension as $\left[\mathrm{HgCl}_{4}\right]^{2-}, \mathrm{HCl}\left(30 \%\right.$ Suprapure $^{\mathrm{TM}}$ grade, Merck) was added to the samples to a final concentration of $0.75 \%$.

\subsubsection{ICP-MS analysis}


The samples were analysed by ICP-MS (7700x, Agilent Technologies, Santa Clara, CA, US), a method widely used to analyse metal content migrating from plastic into various food stimulants (e.g. Dutra et al., 2014; Cheng et al., 2010) and that has previously also been used for analysis of the total metal content in waste plastic (e.g. Götze et al., 2016). Yttrium and Scandium were added directly as internal standards. The instrument was equipped with a platinum-tipped skimmer and sample cones, a double-pass Scott spray chamber operating at $2^{\circ} \mathrm{C}$ and a Micromist spray chamber. The use of Pt-tipped cones, instead of standard Ni-tipped cones, was imperative in order to achieve a stable analysis, as the high acid content and amount of organic material in the samples would degrade the latter and leave a lot of residue around the cone entrance. All elements, except $\mathrm{Al}$ and $\mathrm{Li}$, were analysed in helium collision mode. $\mathrm{Al}$ and Li were analysed with no gas. He flow $(5 \mathrm{~mL} / \mathrm{min})$, carrier gas flow $(0.34 \mathrm{~mL} / \mathrm{min})$, dilution gas flow $(0.59 \mathrm{~mL} / \mathrm{min})$ and sample cone depth $(8 \mathrm{~mm})$ were optimised in order to achieve optimal sensitivity for the elements in the sample matrix.

Recovery rates were monitored by adding the investigated elements to the acid mixture before acid digestion at the 1 and $10 \mu \mathrm{g} / \mathrm{g}$ levels. The method limit of detection (LOD) was calculated as Average Blank $+3 *$ Stdev Blank, and the blank samples consisted of the acid mix and were passed through the microwave digestion procedure together with the samples.

\subsection{Statistical analysis of compositional data}

Analytical results produced in the present work (i.e. metal concentrations) are considered compositional or closed data. This means that the different variables in the dataset are inherently linked to each other (Edjabou et al., 2017). As traditional statistical operations are based on the assumption that the variables are independent, applying traditional statistics to compositional data can provide misleading results (Edjabou et al., 2017). To overcome this potential limitation, transformation of datasets before statistical analyses has been proposed (Edjabou et 
al., 2017; Martín-Fernández et al., 2015). For the purpose of transformation and statistical analysis, undetected values were replaced with $65 \%$ of the LOD, following the approach in Palarea-Albaladejo and Martín-Fernández (2015). However, datasets with a large share of results below the LOD may introduce bias into statistical assessments (Antweiler, 2015; Antweiler and Taylor, 2008). Consequently, metals where more than $50 \%$ of the samples exhibited concentrations below the LOD were excluded from the datasets statistically analysed. This approach was applied to both the total dataset and to the dataset including only the waste samples, as more information was available for the waste samples (separation method, previous use and washing) than for the reprocessed and virgin plastic samples. Details regarding data transformation, software, statistical analyses and p-values are provided in Appendix E1 (SM).

\section{Results and discussion}

\subsection{Metal concentrations}

A summary of analytical results is presented in Table 2 for all analysed metals. Detailed results for each sample are provided inTable B.1 (SM). case. As an example, the highest FOD in this study of $85 \%$ was observed for Fe, which also had one of the highest LODs of $3.40 \mu \mathrm{g} / \mathrm{g}$. Recovery efficiencies are presented in the Table C.1 (SM).

\section{$>$ Table $2<$}

$0.04 \mu \mathrm{g} / \mathrm{g}$ for $\mathrm{Cd}$ and As to $43 \mu \mathrm{g} / \mathrm{g}$ for Al. At the same time, the FODs ranged from $10 \%$ for $\mathrm{Hg}$ to $85 \%$ for Fe and Ti. There is often a negative correlation between LOD and FOD, meaning that the higher the LOD the lower the FOD. However, for several of the metals this was not the 
Concentration ranges of the individual metals varied by almost six orders of magnitude, from the lowest detection limit of $0.04 \mu \mathrm{g} / \mathrm{g}$ for $\mathrm{As}$ and $\mathrm{Cd}$, to the largest measured concentration for $\mathrm{Ti}$ at $13800 \mu \mathrm{g} / \mathrm{g}$. Even the mean concentration of each metal varied by almost four orders of magnitude, from $0.70 \mu \mathrm{g} / \mathrm{g}$ for $\mathrm{Li}$, to $2090 \mu \mathrm{g} / \mathrm{g}$ for $\mathrm{Ti}$, which indicates that the presence of different metals in plastic diverges considerably. Additionally, large variations were observed in most metals, as the differences between the minimum and maximum measured concentrations varied from one to four orders of magnitude for all metals, besidesHg. As an example, the lowest measured Cd concentration was $<0.04 \mu \mathrm{g} / \mathrm{g}$, whereas the highest was 167 $\mu \mathrm{g} / \mathrm{g}$. Thus, the results show that the presence of even a single metal can vary considerably among plastic samples. This clearly demonstrates the complexity of plastic sample chemistry and illustrates that specific chemical data are needed to characterise recyclable plastic and that caution is required when applying such data in other contexts.

The concentrations of all metals, except for $\mathrm{As}, \mathrm{Fe}, \mathrm{Cd}$ and $\mathrm{Ti}$, were within the same order of magnitude as reported in plastic waste samples by Götze et al. (2016), whereas the concentrations of $\mathrm{As}, \mathrm{Fe}, \mathrm{Cd}$ and $\mathrm{Ti}$ were considerably lower. However, the findings in the review by Bach et al. (2008) reported concentrations of Fe as low as $1.3 \mu \mathrm{g} / \mathrm{g}$ in PET mineral water bottles, which is within the same order of magnitude as the lowest Fe concentration measured in this study $(3.40 \mu \mathrm{g} / \mathrm{g})$. The highest measured Cd concentration of $167 \mu \mathrm{g} / \mathrm{g}$ was exceptionally high compared to several other studies (reported in Götze et al., 2016), and it also exceeded the limit value of $0.01 \% \mathrm{Cd}$ in plastic used for electrical and electronic equipment (EU, 2011a). Nevertheless, Burnley (2007) reported Cd concentration in plastic as high as 388 $\mu \mathrm{g} / \mathrm{g}$; moreover, this high concentration was only measured in a single sample of reprocessed PE plastic from HHW (group 2), which is a sample that also showed remarkably high $\mathrm{Pb}$ and Cr concentrations. Considering all of the above, the concentrations measured in this study were found to be within the ranges of the available literature data. 


\subsection{Statistical analysis}

The MANOVA revealed a significant difference ( $\mathrm{p}$-value $<2.2 \cdot 10^{-16}$ ) in concentrations in samples of different polymer types and samples of different origin across all metals included in the statistical analysis. Additionally the analysis showed statistical differences between nonwashed and washed waste plastic samples, with a $\mathrm{p}$-value $=5.82 \cdot 10^{-3}$. This is elaborated on further in the following sections. Detailed results from all statistical analyses are presented in Appendix E (SM).

\subsubsection{All samples: Importance of the polymer type}

In Figure 3, the concentrations of all 15 metals, divided according to polymer types (PET, PE, PP and PS), are illustrated with boxplots. The most distinctive tendency was that of Sb, where PET samples contained considerably higher concentrations when compared to samples of any of the other polymer types. Additionally, Figure 3 shows that the concentrations of $\mathrm{Zn}$ in the PS samples were higher than in samples of PET and PP. This was confirmed by the statistical analysis, as $\mathrm{Sb}$ was the main reason for the difference between PET samples and samples of other polymers, and Zn contributed to the significant difference between PET and PS, as well as PP and PS. Finally, data presented in Figure 3 suggests that the concentrations of Co in PET samples, As and $\mathrm{Cu}$ in PP samples and $\mathrm{Li}$ in PS samples were higher than in the remaining samples; however, these differences were not found statistically significant.

\section{$>$ Figure $3<$}

Diantimony trioxide $\left(\mathrm{Sb}_{2} \mathrm{O}_{3}\right)$ can be used as a catalyst during PET production and is the dominant catalyst for this purpose in Europe (EU, 2008). Even though a catalyst is not supposed 
to end up in the final product, literature data suggest that trace amounts of $\mathrm{Sb}$ can be detected in PET (Bach et al., 2008). Hence, the significantly higher concentrations of Sb in PET samples found in the present study can be explained potentially by contamination during the PET production process. This is further supported by the fact that PET samples from all four sample groups included samples with $\mathrm{Sb}$ concentration $>100 \mu \mathrm{g} / \mathrm{g}$.

Similarly, the high $\mathrm{Zn}$ concentrations in PS samples indicated contamination or intentional addition of $\mathrm{Zn}$ in the production stage (use of $\mathrm{Zn}$-containing catalysts, $\mathrm{Zn}$-containing additives, etc.), since all PS samples (from groups 1, 3 and 4 - no PS samples from sample group 2 was included in the study) exhibited a $\mathrm{Zn}$ concentration $>50 \mathrm{~g} / \mathrm{g}$. This could be related potentially to the use of zinc stearate as a lubricant, as it is employed in the production of highimpact PS (making up to $0.15 \%$ of the final product) (ISE, 2012).

In summary, there are considerable differences between the samples of different polymer types, mainly due to higher concentrations of $\mathrm{Sb}$ in the PET samples and $\mathrm{Zn}$ in the PS samples.

\subsubsection{All samples: Importance of the origin}

In Figure 4, the concentrations of all 15 metals, grouped according to the different sample origins (waste plastic, reprocessed plastic from HHW, reprocessed plastic from IW and virgin plastic), are illustrated with boxplots. The geometric mean in all four sample groups for all metals are presented in Table B.2 (SM).

Overall, the concentration variations (the whiskers in the boxplot illustrate the variation between the $25^{\text {th }}$ and $75^{\text {th }}$ percentile) for all metals were much larger within the waste samples and reprocessed samples from HHW, when compared to variations within the virgin samples. This could be related to household waste being a more heterogeneous material stream (Ragaert et al., 2017), containing many different products with a wide range of additives and 
contaminants. The variations in metal concentrations in reprocessed samples from IW was in general lower than for reprocessed or waste plastic from HHW, albeit they were still higher than for the virgin samples.

\section{$>$ Figure $4<$}

Hotelling's T tests showed a significant difference in the geometric mean between all four groups of origin, across all metals. In all cases, the metal concentrations were lower in the virgin samples compared to the other origins, which is clear from Figure 4, with the exception of $\mathrm{Hg}$, as it was only detected in $10 \%$ of the samples. The Ti concentration was found to be significantly lower in virgin samples compared to samples of the remaining origins.

When compared to virgin plastic, the mechanical properties of reprocessed or recycled plastic are often inferior (van der Harst et al., 2016; Luijsterburg and Goossens, 2014; Vilaplana and Karlsson, 2008), so fillers and additives are commonly added during recycling to improve material quality (Gu et al., 2017). This includes the addition of titanium dioxide $\left(\mathrm{TiO}_{2}\right)$ as filler (Gu et al., 2017), potentially explaining the significantly higher Ti concentrations in the reprocessed plastic samples compared to its virgin counterpart (Figure 4). However, potential $\mathrm{TiO}_{2}$ additions do not explain the high $\mathrm{Ti}$ concentrations in the waste samples, and the reasons for its presence in waste and reprocessed plastic are therefore expected to be manifold. Nevertheless, $\mathrm{TiO}_{2}$ has been classified recently as a suspected carcinogen (ECHA, 2017), raising concerns about its presence in plastic.

Comparing the metal concentration in reprocessed and waste samples from HHW with those in virgin samples, additional metals, besides $\mathrm{Ti}$, contributed to the difference. These included $\mathrm{Al}, \mathrm{Zn}$ and $\mathrm{Pb}$, and additionally $\mathrm{Cr}, \mathrm{Mn}, \mathrm{Co}$ and $\mathrm{Cu}$ for waste samples only (see Figure E.2 (SM)). Their presence could be due to the use of metal-containing additives during 
conversion of virgin raw plastic material into products, a process that both the plastic waste and reprocessed plastic has been through (see Figure 1). However, plastic is a very heterogeneous material and the use of additives therefore depends on several factors, such as polymer type, product type, etc. Even though some knowledge exist in relation to the generic use of additives in plastic (e.g. Hahladakis et al., 2018; Hansen, 2013), very limited information is publically available with respect to the use of specific additives for specific types of products made of specific polymers. Additionally, the metals could originate from contamination during use, metal impurities in non-metal additives, etc. As a result, the possible origins of metals in plastic are numerous and further research is necessary to identify the specific sources of individual metals.

The metal concentrations in reprocessed samples from HHW was significantly higher than in waste samples and reprocessed samples from IW, and were thereby the sample group exhibiting the overall highest metal concentrations. For the waste samples, this was due mainly to the concentrations of $\mathrm{Cu}, \mathrm{Pb}$, and $\mathrm{Zn}$, as these were significantly higher in the reprocessed samples from HHW, as illustrated in Figure 4. Lower concentrations in reprocessed plastic from IW compared to HHW confirms that IW is a more homogeneous and often less contaminated waste stream than HHW (Ragaert et al., 2017), while higher concentrations in reprocessed plastic from HHW, compared to plastic waste from households, could suggest the use of metalcontaining additives (zinc oxide, titanium dioxide, metal powder as fillers, metal salts as heat stabilisers (Hahladakis et al., 2018; Gu et al., 2017), etc.) in the recycling process.

Overall, the results presented in this section suggest that virgin plastic generally contains lower metal concentrations when compared to reprocessed plastic and un-processed plastic waste.

\subsubsection{Waste samples: Importance of collection, previous application and washing}


Difference between waste samples was statistically analysed, including differences between collection schemes (source separation [sample group 1a] and residual [sample group 1b]), previous applications (food contact [sample groups 1a.1 and 1a.2] and non-food contact items [sample groups 1a.3 and 1a.4]) and treatment (non-washed [sample group 1a.1, 1a.3 and 1b.1] and washed [sample group 1a.2, 1a.4 and 1b.2]).

The differences between metal concentrations in samples from source-separated and residual plastic waste were insignificant (p-value of 0.13 ). Similarly, there was no significant difference in metal concentration between samples of food and non-food contact items ( $p$-value of 0.18 ). As the legislation related to food packaging is very comprehensive (including migration limits for more than 800 substances), it was expected that products approved for food contact would contain lower concentrations of metals, compared to non-food contact items. However, the insignificant difference could be due to several aspects. First, the legislation only includes migration limits. Metals used in additives are assumed to be bound solidly in the plastic and therefore expected to be released primarily by wear and tear and not migration (Hansen, 2013). Hence, the effects of migration limits on the chemical composition of plastic are expected to be more evident for organic substances prone to migration. Second, non-food plastic items from Europe might be produced in many cases from food-grade plastic, even though this is not legally a necessity.

On the other hand, there was a significant difference (p-value of 0.005 ) in metal concentrations between non-washed and washed samples. Table 3 presents the geometric mean for all waste samples, as well as for the non-washed and the washed samples, for all the metals. For most metals ( $\mathrm{Al}, \mathrm{As}, \mathrm{Cr}, \mathrm{Cu}, \mathrm{Fe}, \mathrm{Li}, \mathrm{Mn}, \mathrm{Ni}, \mathrm{Pb}$ and $\mathrm{Zn}$ ), the geometric mean of the nonwashed samples was higher than for the washed samples, whereas for the remaining metals $(\mathrm{Cd}$, $\mathrm{Co}, \mathrm{Hg}, \mathrm{Sb}$ and $\mathrm{Ti}$ ) it was lower. However, the bootstrap analysis showed that this difference was only significant for Mn (see Figure E.3 (SM)). Consequently, the difference in these metals 
between non-washed and washed samples cannot be explained by physical contamination that may easily be removed from the plastic surface through washing, except in the case of Mn.

It should be noted that some of the $\mathrm{Fe}$ and $\mathrm{Cr}$ found in the waste samples could have originated from the shredding equipment used during the pre-treatment of the waste samples, as it was made of stainless steel (containing $\mathrm{Fe}$ and $\mathrm{Cr}$ ). This notion is supported by Romão et al. (2010), who found that $\mathrm{Fe}$ is an intrinsic contaminant after recycling bottle-grade PET, originating from steel and abrasion during the cleaning process. However, as the difference in $\mathrm{Fe}$ and $\mathrm{Cr}$ concentrations between washed and non-washed samples was not significant, indicating that $\mathrm{Fe}$ and $\mathrm{Cr}$ were not present as physical impurities, contamination from processing equipment was expected to be limited in the present study.

\subsection{Plastic quality and recycling}

As previously demonstrated, the samples of reprocessed plastic from HHW contained higher concentrations of metals than virgin plastic. This potentially affects the quality - and applicability - of reprocessed plastic, as it might be unsuitable for use in certain applications (e.g. food packaging), due to elevated metal concentrations.

In Europe, the legislation related to using plastic in food contact items is the most comprehensive in regards to chemical content, followed by legislation for use in toys. These legislations include migration limits for a wide range of selected substances, including metals, but no limits for the total content of metals in plastic. As it is unknown if and how much the metals could potentially migrate from plastic, a direct comparison of results from this study to legal migration limits is not possible. Instead, the metal concentrations from this study are in Figure 5 compared to the US legal limit values for $\mathrm{Pb}$ and $\mathrm{Cd}$ in toys (Guney and Zagury, 2012) 
as well as concentrations of $\mathrm{Cr}, \mathrm{Mn}, \mathrm{Fe}$ and $\mathrm{Co}$ previously found in plastic food packaging

514 (Bach et al., 2008; Romão et al., 2010). Moreover, the metal concentrations are compared to the European legal limit value for $\mathrm{Cr}, \mathrm{Cd}, \mathrm{Hg}$ and $\mathrm{Pb}$ in electrical and electronic equipment (EU, 2011a) and the European limit values for Cd that dictates whether or not a reprocessed plastic can be placed on the European market at all (EU, 2006). equipment and all European plastic) was exceeded for reprocessed plastic from HHW, albeit only by a single PE sample. Similarly, the limit values for use in toys were exceeded for Pb by the same sample of reprocessed PE from HHW. Although all samples of secondary plastic used in this study showed metal concentrations higher than that of virgin plastic, all of them (except for the single PE sample) could be used in electrical and electronic equipment as well as toys. However, the results clearly illustrate the extent of metal content variation and the challenge of controlling the presence of metals in reprocessed plastic derived from HHW. This could particularly be an issue for metals like $\mathrm{Cd}$, where exceeding the EU limit results in it being banned for use in any plastic product on the European market. Such uncertainty in the quality of reprocessed plastic from HHW limits its applicability and use.

As the direct comparison of metal concentrations from this study and legal limit values for food-contact items was challenging, the results were compared to concentrations of $\mathrm{Cr}, \mathrm{Fe}$,

534 Mn and Co reported in the literature for food contact items (Bach et al., 2008; Romão et al., 2010). The $\mathrm{Cr}, \mathrm{Fe}$ and $\mathrm{Mn}$ concentrations corresponded to the lower concentrations measured in this study (Figure 5), while $\mathrm{Cr}$ and $\mathrm{Fe}$ concentrations from the literature were lower than 
$\mathrm{Cr}$ ) and $57 \%$ (for $\mathrm{Fe}$ ) of samples were below LOD. Mn concentrations from the literature were lower than in any of the reprocessed samples from HHW, while Co concentrations in food packaging corresponded to the highest concentrations measured in waste samples in this study. Nonetheless, plastic approved for food contact may contain substantially lower metal concentrations when compared to results for reprocessed plastics from HHW in this study (e.g. $\mathrm{Cr}$ and $\mathrm{Fe}$ - Figure 5). Consequently, as far as metals are concerned, recycled plastics may be unsuitable for food packaging applications.

Although reprocessed plastic from HHW may comply with the regulatory limits at the first recycling cycle, the elevated metal concentrations and the large variety amongst reprocessed plastic from HHW might still reduce the suitability of the recycled plastic for certain applications and thereby limit the applicability indirectly. The European recycling rate for plastic packaging was $40.3 \%$ in 2015 (Eurostat, 2018). If the $55 \%$ recycling rate is to be met in 2030 (EC, 2018b) much more plastic needs to be recycled, potentially some plastic that has been recycled before. Lack of metal removal and the addition of metal-containing additives during plastic recycling may thereby lead to accumulation of metals due to increased recycling rates. A similar trend has been quantitatively illustrated with the example of selected organic substances in paper, a material with high recycling rates (Pivnenko et al., 2016a). Depending on the form of the metals in plastic, elevated concentrations might also pose a risk to human health and the environment. Consequently, an analysis of the form and species of metals in plastic and the mode of their release is a natural and important continuation of the work as support for future risk assessment studies. Consequently, even though the elevated concentration of metals in reprocessed plastic currently may not limit applicability directly, it is important to be aware that potentially hazardous metals are present in reprocessed and recycled plastic. 


\section{Conclusion}

564 Eighty-two plastic samples, including waste plastic from households, reprocessed plastic waste from households and industry, and virgin plastic, were obtained and analysed for 15 selected metals. The metal concentrations varied considerably, across different metals (from $<0.04 \mu \mathrm{g} / \mathrm{g}$ for $\mathrm{Cd}$ and As to $13800 \mu \mathrm{g} / \mathrm{g}$ for Ti) and samples (e.g. from $3.90 \mu \mathrm{g} / \mathrm{g}$ to $13800 \mu \mathrm{g} / \mathrm{g}$ for Ti). The statistical analysis showed that both polymer type and origin of the plastic samples were determining factors for the observed differences in metal concentrations. The difference between polymer types was dominated by significantly higher Sb concentrations in PET samples, and higher Zn concentrations in PS samples when compared to PET and PP samples. Plastic samples from households (both waste and reprocessed plastic samples) contained significantly higher concentrations of $\mathrm{Al}, \mathrm{Pb}, \mathrm{Ti}$, and $\mathrm{Zn}$, when compared to virgin plastic samples. Moreover, samples of reprocessed HHW contained the overall highest concentrations of metals, potentially related to the use of metal-containing additives (e.g. fillers) to enhance the mechanical properties of plastic during reprocessing. The differences in metal concentrations between waste samples appeared to be associated with differences in material chemistry rather than physical contamination, as washing could not explain the differences (except for Mn). The elevated metal concentrations in reprocessed samples from household waste did not exceed the few legal limit values currently available, with the exception of a single reprocessed PE sample from HHW. However, as some metals are potentially harmful and toxic, it is important to be aware that concentrations are higher in reprocessed plastic from household waste and that a continuous increase in plastic recycling rates may lead to even higher metal concentrations in the future.

\section{Acknowledgement}

This work was supported by the Danish Environmental Protection Agency (Miljøstyrelsen). 


\section{References}

590

591

Antweiler, R.R., Taylor, H.E., 2008. Evaluation of Statistical Treatments of Left-Censored Environmental Data using Coincident Uncensored Data Sets: I. Summary Statistics. Environmental Science and Technology. https://doi.org/10.1021/es071301c

Antweiler, R.C., 2015. Evaluation of Statistical Treatments of Left-Censored Environmental Data Using Coincident Uncensored Data Sets. II. Group Comparisons. Environmental science and technology. https://doi.org/10.1021/acs.est.5b02385

Bach, C., Dauchy, X., Chagnon, M., Etienne, S., 2012. Chemical compounds and toxicological assessments of drinking water stored in polyethylene terephthalate (PET) bottles: A source of controversy reviewed. Water Research. https://doi.org/10.1016/j.watres.2011.11.062

Boldrin, A., Christensen, T.H., 2010. Seasonal generation and composition of garden waste in Aarhus (Denmark). Waste Mangement. https://doi.org/10.1016/j.wasman.2009.11.031

Burnley, S., 2007. The use of chemical composition data in waste management planning - A case study. Waste Management. https://doi.org/10.1016/j.wasman.2005.12.020

Camacho, W., Karlsson, S., 2001. Quality-determination of recycled plastic packaging waste by identication of contaminants by GC \pm MS after microwave assisted extraction (MAE). Polymer degradation and stability. https://doi.org/10.1016/S0141$\underline{3910(00) 00163-4}$

Cheng, X., Shi, H., Adams, C.D., Ma, Y., 2010. Assessment of metal contaminations leaching out from recycling plastic bottles upon treatments. Environmental Science and Pollution Research. https://doi.org/10.1007/s11356-010-0312-4

Cossu, R., Lai, T., Pivnenko, K., 2012. Waste washing pre-treatment of municipal and special waste. Journal of hazardous materials. https://doi.org/10.1016/j.jhazmat.2011.07.121 
Dahlén, L., Lagerkvist, A., 2008. Methods for household waste composition studies. Waste Management. https://doi.org/10.1016/j.wasman.2007.08.014

Dutra, C., Freire, M.T.A., Nerín, C., Bentayeb, K., Rodriguez-Lafuente, A., Aznar, M., Reyes, F.G.R., 2014. Migration of residual nonvolatile and inorganic compounds from recycled post-consumer PET and HDPE. Journal of the Brazilian Chemical Society. https://doi.org/10.5935/0103-5053.20140016

EC (European Commission), 2004. Regulation (EC) No 1935/2004 of the European Parliament and of the Council on materials and articles intended to come into contact with food and repealing Directives 80/590/EEC and 89/109/EEC. Official Journal of the European Union. L338, 4-17.

EC (European Commission), 2008. Regulation (EC) No 1272/2008 of the European Parliament and of the council on classification, labelling and packaging of substances and mixtures, amending and repealing Directives 67/548/EEC and 1999/45/EC, and amending Regulation (EC) No 1907/2006. Official Journal of the European Union. L353, 1-1355.

EC (European Commission), 2015. Communication from the commission to the European Parliament, the Council, the European Economic and Social Committee of the committee of the regions: Closing the loop - An EU action plan for the Circular Economy. Brussels, 2.12.2015.

EC (European Commission), 2018a. Communication from the commission to the European Parliament, the Council, the European Economic and Social Committee of the regions: A European Strategy for Plastics in a Circular Economy. Brussels, 16.1.2018.

EC (European Commission), 2018b. DIRECTIVE (EU) 2018/... OF THE EUROPEAN PARLIAMENT AND OF THE COUNCIL of ... amending Directive 94/62/EC on packaging and packaging waste. ANNEX. 
ECHA (European Chemical Agency), 2017. Titanium dioxide proposed to be classified as suspected of causing cancer when inhaled. https://echa.europa.eu/da/-/titaniumdioxide-proposed-to-be-classified-as-suspected-of-causing-cancer-when-inhaled $[11 / 01 / 2018]$

Edjabou, M.E., Jensen, M.B., Götze, R., Pivnenko, K., Petersen, C., Scheutz, C., Astrup, T.F., 2015. Municipal solid waste composition: Sampling methodology, statistical analyses, and case study evaluation. Waste Management. https://doi.org/10.1016/j.wasman.2014.11.009

Edjabou, M.E., Martín-Fernández, J.A., Scheutz, C., Astrup, T.F., 2017. Statistical analysis of solid waste composition data: Arithmetic mean, standard deviation and correlation coefficients. Waste Management. https://doi.org/10.1016/j.wasman.2017.08.036

Ellen MacArthur Foundation, 2016. The New Plastics Economy: Rethinking the future of plastics [online report]. https://www.ellenmacarthurfoundation.org/assets/downloads/EllenMacArthurFoundati on_TheNewPlasticsEconomy_15-3-16.pdf [08/05/2017]

Eriksen, M.K., Damgaard, A., Boldrin, A., Astrup, T.F., 2018. Quality assessment and substitutability of recycled plastic from household waste. Submitted

EU (European Union), 2006. Regulation (EC) No 1907/2006 of the European Parliament and of the Council concerning the Registration, Evaluation, Authorisation and Restriction of Chemicals (REACH), establishing a European Chemicals Agency, amending Directive 1999/45/EC and repealing Council Regulation (EEC) No 793/93 and Commission Regulation (EC) No 1488/94 as well as Council Direc- tive 76/769/EEC and Commission Directives 91/155/EEC, 93/67/EEC, 93/105/EC and 2000/21/EC. Official Journal of the European Union. L136, 3-280. 
EU (European Union), 2008. Risk Assessment Report: Diantimony Trioxide. CAS No: 130964-4, EINECS No: 215-175-0. Sweden, May 2008.

EU (European Union), 2011a. Directive 2011/65/EU of the European Parliament and of the Council on the restriction of the use of certain hazardous substances in electrical and electronic equipment. Official Journal of the European Union. L174, 88-110.

EU (European Union), 2011b. Commission regulation (EU) No 2011/10 on plastic materials and articles intended to come into contact with food. Official Journal of the European Union. L12, 1-89.

Eurostat, 2018. Recycling rate of packaging waste by type of packaging. http://ec.europa.eu/eurostat/tgm/refreshTableAction.do?tab=table\&plugin $=1 \&$ pcode $=\mathrm{c}$ ei_wm020\&language=en $[11 / 04 / 2018]$.

Goldberg, 2017. What about the Circularity of Hazardous Materials? Journal of Industrial Ecology. https://doi.org/10.1111/jiec.12585

Government of Japan, 2010. Establishing a sound material-cycle society - Milestone toward a sound material-cycle society through changes in business and life styles. Ministry of the Environment.

Götze, R., Pivnenko, K., Boldrin, A., Scheutz, C., Astrup, T.F., 2016. Physico-chemical characterisation of material fractions in residual and source-segregated household waste in Denmark. Waste Mangement. https://doi.org/10.1016/j.wasman.2016.05.009

Gu, F., Guo, J., Zhang, W., Summers, P.A., Hall, P., 2017. From waste plastics to industrial raw materials: A life cycle assessment of mechanical plastic recycling practice based on a real-world case study. Science of the total environment.

\section{https://doi.org/10.1016/j.scitotenv.2017.05.278}


Guney, M., Zagury, G.J., 2012. Heavy metals in toys and low-cost jewelry: Critical review of U.S. and Canadian legislations and recommendations for testing. Environmental Science and Technology. https://doi.org/10.1021/es203470x

Hahladakis, J.N., Velis, C.A., Weber, R., Iacovidou, E., Purnell, P., 2018. An overview of chemical additives present in plastics: Migration, release, fate and environmental impact during their use, disposal and recycling. Journal of Hazardous Materials. https://doi.org/10.1016/j.jhazmat.2017.10.014

Hansen, E., 2013. Hazardous substances in plastic materials. Report by COWI and Danish Technological Institute on behalf of the Norwegian Environment Agency.

Huber, M., Franz, R., 1997. Identification of Migratable Substances in Recycled High Density Polyethylene Collected from Household Waste. Journal of High Resolution Chromatography. https://doi.org/10.1002/jhrc.1240200806

ISE (Inclusive Science and Engineering), 2012. High Impact Polystyrene Manufacturing Process [website]. Available at: http://www.inclusive-science-engineering.com/highimpact-polystyrene-manufacturing-process/ [November 06 2017]

Lagerkvist, A., Ecke, H., Christensen, T.H., 2011. Chapter 2.1 - Waste Characterization: Approaches and Methods in Solid Waste Technology \& Management. Blackwell Publishing Ltd. ISBN: 978-1-405-17517-3.

Lahimer, M.C., Ayed, N., Horriche, J., Belgaied, S., 2013. Characterization of plastic packaging additives: Food contact, stability and toxicity. Arabian Journal of Chemistry. https://doi.org/10.1016/j.arabjc.2013.07.022

Luijsterburg, B., Goossens, H., 2014. Assessment of plastic packaging waste: Material origin, methods and properties. Resource, Conservation and Recycling, vol. 85, pp. 88-97. https://doi.org/10.1016/j.resconrec.2013.10.010 
Martín-Fernández, J., Daunis-i-Estadella, J., Mateu-Figueras, G., 2015. On the interpretation of differences between groups for compositional data. Sort-statistics and Operations Research Transactions. Volume 39(2), 231-252.

Nederskov, J., 2017. Personal communication. Environmental executive at Amager Ressource Center.

Palarea-Albaladejo, J., Martín-Fernández, J.A., 2015. ZCompositions - R package for multivariate imputation of left-censored data under a compositional approach. Chemometrics and Intelligent Laboratory Systems. https://doi.org/10.1016/j.chemolab.2015.02.019

Pivnenko, K., Astrup, T. F., 2016. The challenge of chemicals in material lifecycles. Waste Management, https://doi.org/10.1016/j.wasman.2016.08.016

Pivnenko, K., Laner, D., Astrup, T.F., 2016a. Material Cycles and Chemicals: Dynamic Material Flow Analysis of Contaminants in Paper Recycling. Environmental Science and Technology. https://doi.org/10.1021/acs.est.6b01791

Pivnenko, K., Eriksen, M.K., Martín-Fernández, J.A., Astrup, T.F., 2016b. Recycling of plastic waste: Presence of phthalates in plastics from households and industry. Waste Management. https://doi.org/10.1016/j.wasman.2016.05.014

PlasticsEurope, EPRO (European Association of Plastics Recycling and Recovery Organisation), 2017. Plastics - the facts [online document]. https://www.plasticseurope.org/application/files/5715/1717/4180/Plastics_the_facts_2 017_FINAL_for_website_one_page.pdf [10/04/2018]

Ragaert, K., Delva, L., Geem, K., 2017. Mechanical and chemical recycling of solid plastic waste. Waste Management. https://doi.org/10.1016/j.wasman.2017.07.044 
Romão, W., Franco, M. F., Bueno, M.I.M.S., Eberlin, M.N., De Paoli, M., 2010. Analysing Metals in Bottle-Grade Poly(ethylene terephthalate) by X-ray Fluorescence Spectrometry. Journal of Applied Polymer Science. https://doi.org/10.1002/app.32232

US EPA (Environmental Protection Agency), 2002. RCRA Waste Sampling Draft Technical Guidance Planning, Implementation, and Assessment.

van der Harst, E., Potting, J., Kroeze, C., 2016. Comparison of different methods to include recycling in LCAs of aluminium cans and disposable polystyrene cups. Waste management. https://doi.org/10.1016/j.wasman.2015.09.027

Vilaplana, F., Karlsson, S., 2008. Quality concepts for the improved use of recycled polymeric materials: A review. Macromolecular Materials and Engineering. https://doi.org/10.1002/mame.200700393

Whitt, M., Brown, W., Danes, J.E., Vorst, K.L., 2016. Migration of heavy metals from recycled polyethylene terephthalate during storage and microwave heating. Journal of plastic film and sheeting. https://doi.org/10.1177/8756087915590190

Winther, J., 2017. Personal communication. Operation coordinator at RenoNord sorting facility. 
749 Table 1: Overview of the plastic samples collected and analysed in the present work.

\begin{tabular}{|c|c|c|c|c|c|c|c|}
\hline \multirow{2}{*}{ Sample group } & \multirow{2}{*}{ Sample shape } & \multirow{2}{*}{ Origin } & \multicolumn{5}{|c|}{ Number of samples } \\
\hline & & & PET & PE & PP & PS & Total \\
\hline \multicolumn{3}{|c|}{ 1a. Source-separated plastic waste from households } & 8 & 8 & 8 & 4 & 28 \\
\hline \multicolumn{3}{|l|}{ 1a.1 Non-washed, food contact } & 2 & 2 & 2 & 1 & 7 \\
\hline 1a.2 Washed, food contact & \multirow{3}{*}{$\begin{array}{l}\text { Plastic flakes } \\
<10 \mathrm{~mm} \text { after } \\
\text { pre-treatment }\end{array}$} & \multirow{3}{*}{$\begin{array}{l}\text { Mechanically sorted } \\
\text { source separated } \\
\text { plastic from HHW }\end{array}$} & 2 & 2 & 2 & 1 & 7 \\
\hline 1a.3 Non-washed, non-food contact & & & 2 & 2 & 2 & 1 & 7 \\
\hline 1a.4 Washed, non-food contact & & & 2 & 2 & 2 & 1 & 7 \\
\hline \multicolumn{3}{|l|}{ 1b. Plastic from residual household waste } & 2 & 2 & 2 & 2 & 8 \\
\hline 1b.1 Non-washed & \multirow{2}{*}{$\begin{array}{l}\text { Plastic flakes } \\
<10 \mathrm{~mm} \text { after } \\
\text { pre-treatment }\end{array}$} & \multirow{2}{*}{$\begin{array}{l}\text { Manually sorted } \\
\text { plastic from residual } \\
\text { HHW }\end{array}$} & 1 & 1 & 1 & 1 & 4 \\
\hline 1b.2 Washed & & & 1 & 1 & 1 & 1 & 4 \\
\hline $\begin{array}{l}\text { 2. Reprocessed plastic from } \\
\text { household waste }\end{array}$ & $\begin{array}{l}\text { Flakes or } \\
\text { pellets }\end{array}$ & $\begin{array}{l}\text { Post-consumer } \\
\text { plastic fully or } \\
\text { partially from } \mathrm{HHW}\end{array}$ & 2 & 5 & 3 & $\mathbf{0}$ & 10 \\
\hline $\begin{array}{l}\text { 3. Reprocessed plastic from } \\
\text { industrial waste }\end{array}$ & $\begin{array}{l}\text { Flakes or } \\
\text { pellets }\end{array}$ & $\begin{array}{l}\text { Plastic waste from } \\
\text { industries, often } \\
\text { pre-consumer }\end{array}$ & 2 & 11 & 3 & 3 & 19 \\
\hline 4. Virgin plastic & $\begin{array}{l}\text { Flakes or } \\
\text { pellets }\end{array}$ & $\begin{array}{l}\text { Virgin } \\
\text { petrochemicals }\end{array}$ & 1 & 8 & 4 & 4 & 17 \\
\hline Total & & & 15 & 34 & 20 & 13 & 82 \\
\hline
\end{tabular}


Table 2: Summary of metal concentrations, limit of detection (LOD) and frequency of detection

753 (FOD) for all metals and all samples. The metal concentrations of each sample were calculated

754 as the geometric mean of the triplicates. Metals with FOD $<50 \%$ were excluded from the

755 statistical analyses (indicated with ${ }^{1}$ for all data and ${ }^{2}$ for waste data)

\begin{tabular}{l|ccccc|cc}
\hline \multirow{2}{*}{ Metal } & Minimum & Median & $\begin{array}{c}\text { Arithmetic } \\
\text { mean }\end{array}$ & $\begin{array}{c}\text { Geometric } \\
\text { mean }\end{array}$ & Maximum & LOD & FOD \\
& $\mu \mathrm{g} / \mathrm{g}$ & $\mu \mathrm{g} / \mathrm{g}$ & $\mu \mathrm{g} / \mathrm{g}$ & $\mu \mathrm{g} / \mathrm{g}$ & $\mu \mathrm{g} / \mathrm{g}$ & $\mu \mathrm{g} / \mathrm{g}$ & $\%$ \\
\hline $\mathrm{Al}$ & $<43.0$ & 160 & 269 & 145 & 2520 & 43.0 & 79 \\
$\mathrm{As}^{1}$ & $<0.04$ & 0.04 & 0.11 & 0.06 & 0.68 & 0.04 & 49 \\
$\mathrm{Cd}^{1,2}$ & $<0.04$ & 0.03 & 2.52 & 0.06 & 167 & 0.04 & 26 \\
$\mathrm{Co}$ & $<0.28$ & 0.63 & 2.47 & 0.79 & 30.9 & 0.28 & 60 \\
$\mathrm{Cr}$ & $<0.72$ & 1.44 & 6.59 & 1.92 & 193 & 0.72 & 62 \\
$\mathrm{Cu}$ & $<0.98$ & 1.90 & 7.71 & 2.49 & 173 & 0.98 & 54 \\
$\mathrm{Fe}$ & $<3.40$ & 52.7 & 158 & 39.4 & 2210 & 3.40 & 85 \\
$\mathrm{Hg}{ }^{1,2}$ & $<1.77$ & 1.17 & 1.26 & 1.25 & 2.01 & 1.77 & 10 \\
$\mathrm{Li}^{1}$ & $<0.21$ & 0.21 & 0.70 & 0.29 & 13.9 & 0.21 & 49 \\
$\mathrm{Mn}$ & $<0.20$ & 1.36 & 2.63 & 0.94 & 56.7 & 0.20 & 72 \\
$\mathrm{Ni}{ }^{1}$ & $<0.88$ & 0.89 & 1.84 & 1.14 & 17.5 & 0.88 & 47 \\
$\mathrm{~Pb}$ & $<0.19$ & 0.26 & 3.39 & 0.47 & 117 & 0.19 & 57 \\
$\mathrm{Sb}$ & $<0.14$ & 0.40 & 34.3 & 1.23 & 304 & 0.14 & 60 \\
$\mathrm{Ti}^{1}$ & $<3.90$ & 1330 & 2090 & 281 & 13800 & 3.90 & 85 \\
$\mathrm{Zn}^{2}$ & $<10.0$ & 13.5 & 35.1 & 19.1 & 186 & 10.0 & 52 \\
\hline
\end{tabular}

Table 3: Geometric mean of all, non-washed and washed waste samples. All values are in $\mu \mathrm{g} / \mathrm{g}$.

The colour indicates the group with geometric mean that is: $\square=$ highest, $\square=$ lowest. Metals

759 with FOD $<50 \%$ were excluded from the statistical analysis (indicated with ${ }^{1}$ ). Significant

760 difference between groups is indicated with ${ }^{2}$.

\begin{tabular}{l|c|c|c|c|c|c|c|c|c|c|c|c|c|c|c}
\hline Geometric mean of: & $\mathbf{A l}$ & $\mathbf{A s}$ & $\mathbf{C d}^{1}$ & $\mathbf{C o}$ & $\mathbf{C r}$ & $\mathbf{C u}$ & $\mathbf{F e}$ & $\mathbf{H g}^{1}$ & $\mathrm{Li}$ & $\mathbf{M n}^{2}$ & $\mathbf{N i}$ & $\mathbf{P b}$ & $\mathbf{S b}$ & $\mathbf{T i}$ & $\mathbf{Z n}^{1}$ \\
\hline All waste samples & 293 & 0.12 & 0.05 & 1.90 & 4.76 & 4.68 & 161 & 1.27 & 0.42 & 2.25 & 1.58 & 0.59 & 3.72 & 2197 & 17.4 \\
\hline Non-washed samples & 373 & 0.13 & 0.05 & 1.71 & 7.06 & 6.63 & 254 & 1.22 & 0.52 & 3.82 & 1.65 & 0.93 & 3.69 & 1880 & 18.5 \\
Washed samples & 231 & 0.11 & 0.05 & 2.12 & 3.20 & 3.30 & 103 & 1.32 & 0.35 & 1.33 & 1.52 & 0.38 & 3.74 & 2567 & 16.3 \\
\hline
\end{tabular}




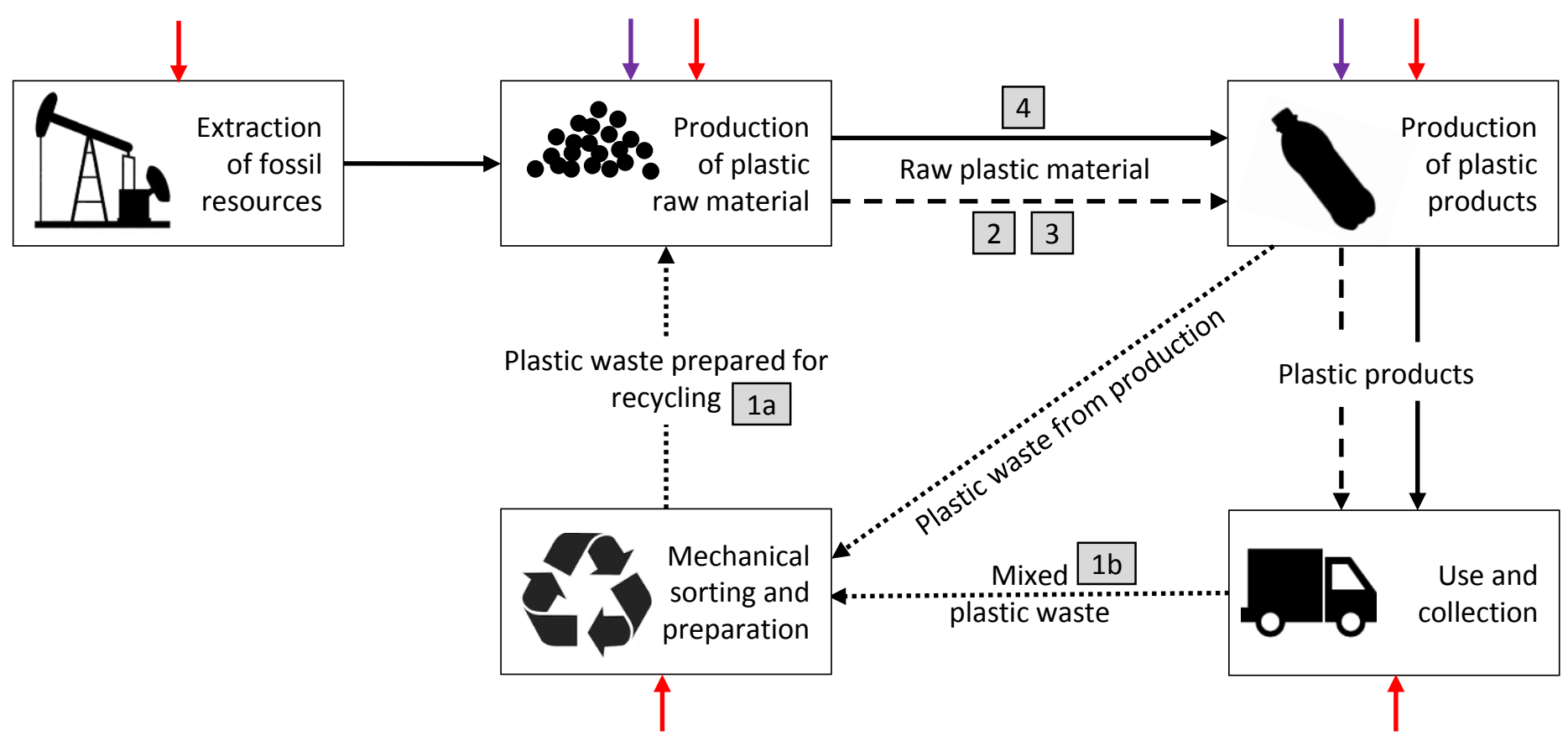
$\longrightarrow$ Flows of virgin plastic
$\longrightarrow$ Intentional use of additives
- - Flows of secondary plastic
Potential contamination
Flows of waste plastic
x Samples collected for analysis. $x$ indicates number of sample group 
Figure 1: Schematic overview of plastic production and recycling. It is indicated from where in the recycling chain the samples have been collected (numbers refer to those given in Table $1)$. 


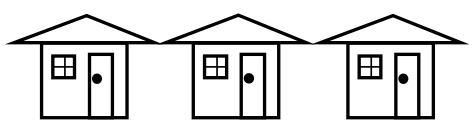

Source segregation

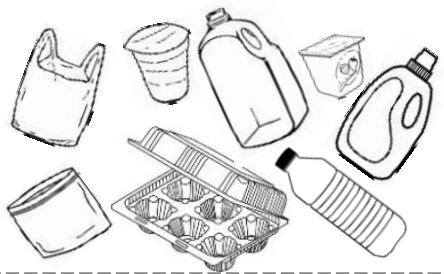

Plastic

waste

$(700 \mathrm{~kg})$
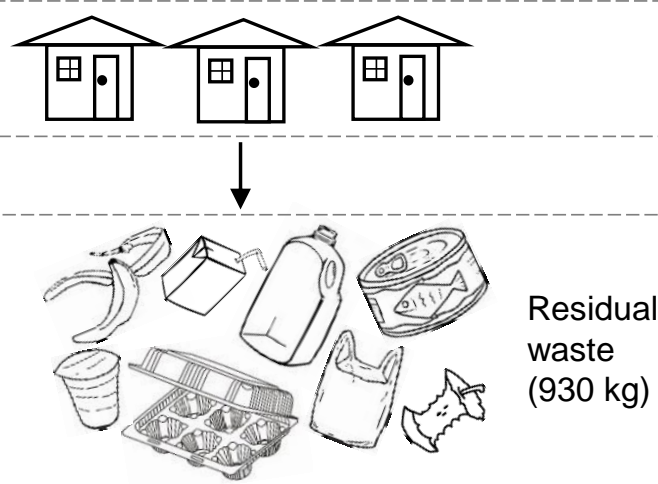

Mechanical sorting
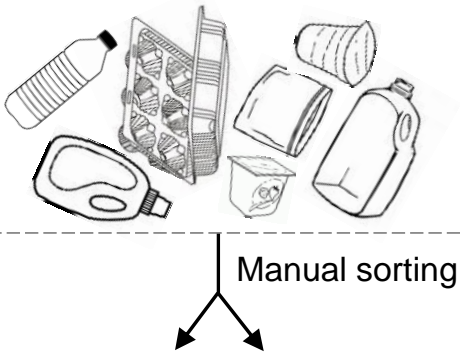

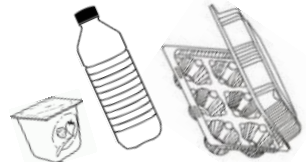

Food contact items
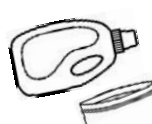

Non-food contact items

Shredding

Shredding

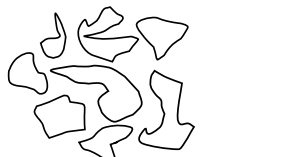

Size reduction*:
"1D splitting"

$\nabla 2 \approx 1 \mathrm{~kg}$

Shredding

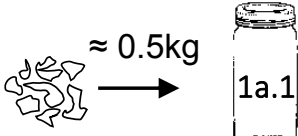

Washing

$\Rightarrow$

$1 \mathrm{a} .2 \approx 0.5 \mathrm{~kg}$

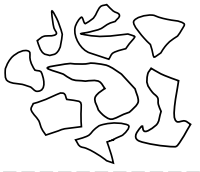

"1D splitting"

Shredding

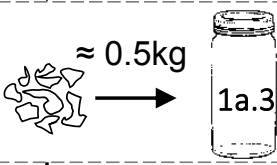

Washing

$\Rightarrow$

$1 \mathrm{a} .4 \approx 0.5 \mathrm{~kg}$

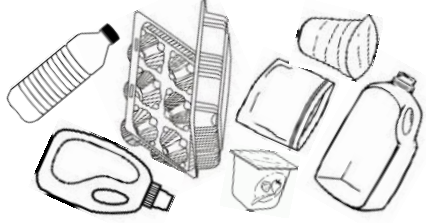

Size reduction:

"Simple random sampling"

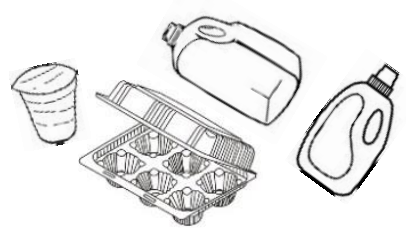

$\downarrow$ Shredding

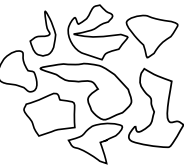

Coarsely shredded plastic

Coarsely shredded plastic

\section{$\downarrow$ Shredding}

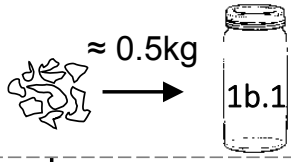

Fine shredded plastic, $<10 \mathrm{~mm}$

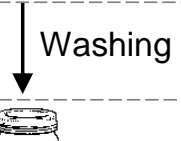

$1 \mathrm{~b} .2 \approx 0.5 \mathrm{~kg}$

Washed plastic, $<10 \mathrm{~mm}$ 
Figure 2: Schematic illustration of the sampling and pre-treatment steps of plastic from household waste, corresponding to samples in group 1a (1a.1-1a.4) and 1b (1b.1-1b.2). * $1 \mathrm{D}$ splitting was only performed on samples with a size $>1.5 \mathrm{~kg}$. 
As

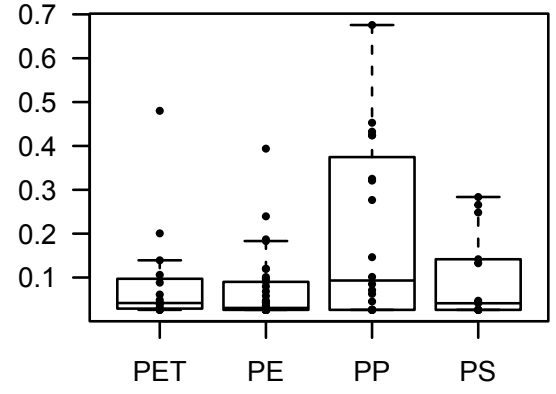

$\mathrm{Cr}$

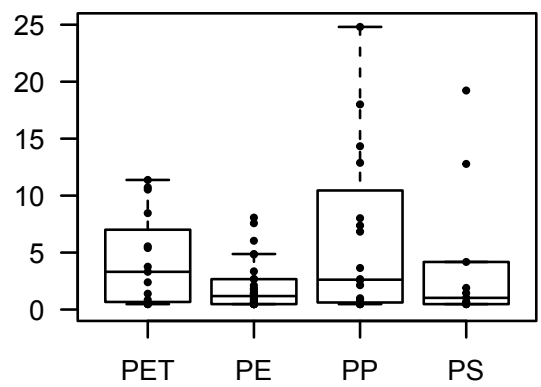

$\mathrm{Hg}$

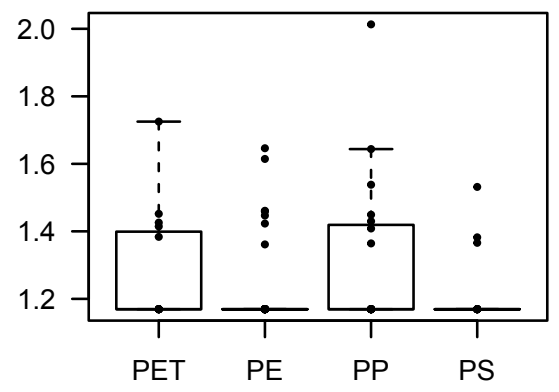

$\mathbf{N i}$

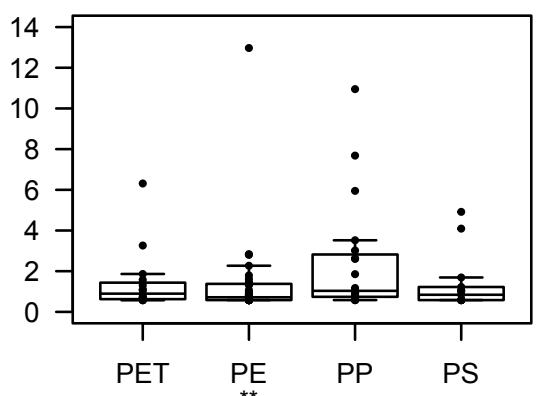

$\mathrm{Ti}$

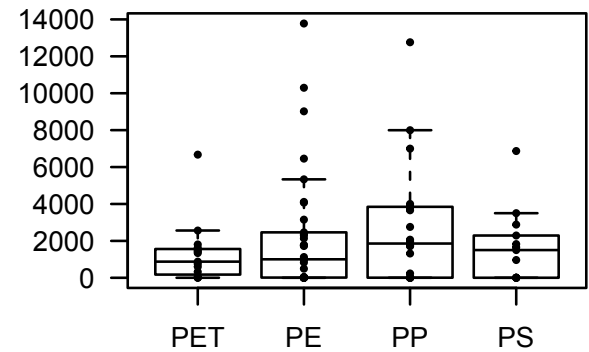

Polymer

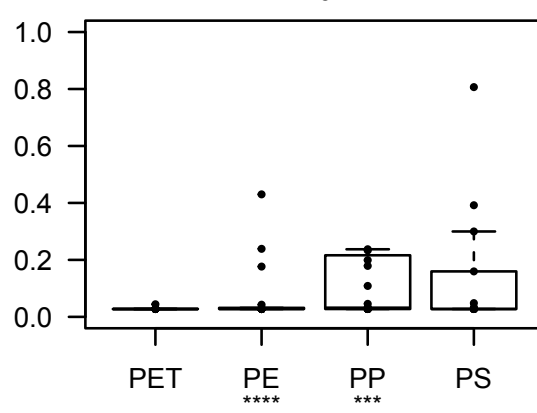

$\mathrm{Cu}$

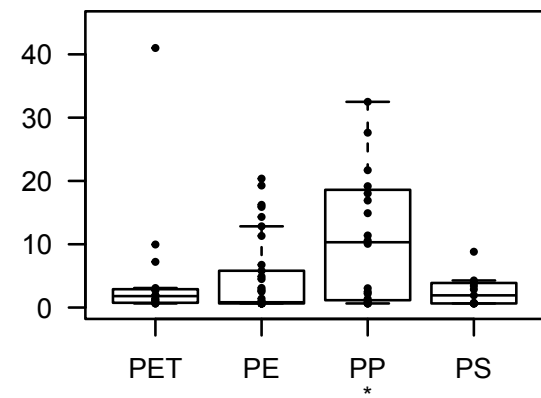

Li

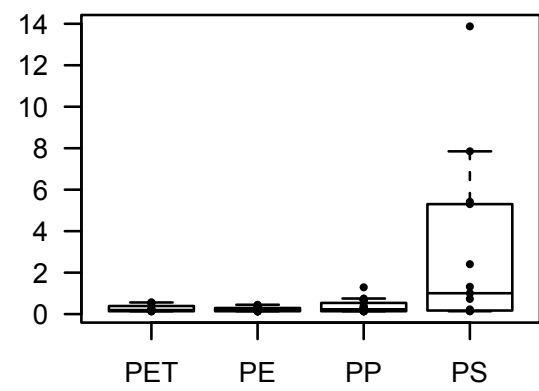

$\mathrm{Pb}$

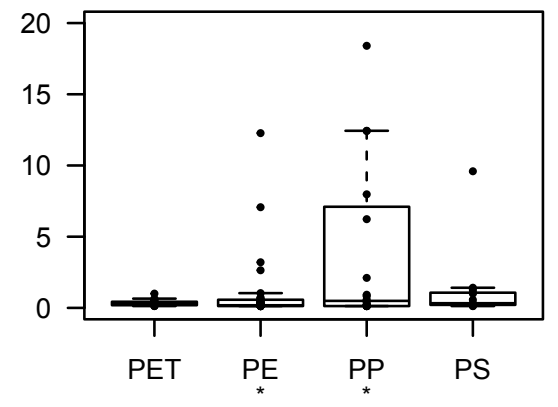

$\mathrm{Zn}$

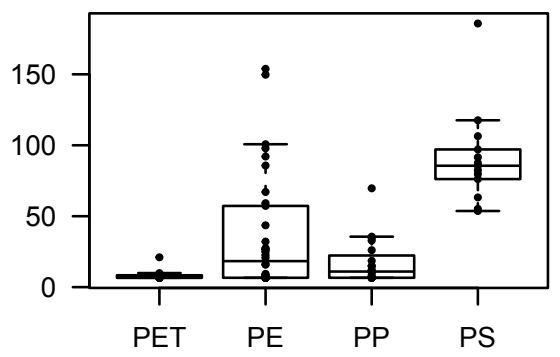

Polymer 
Figure 3: Concentration of 15 metals $[\mu \mathrm{g} / \mathrm{g}]$ based on the polymer type of the samples, illustrated with boxplots. At the boxplot: Lowest whisker = minimum concentration, bottom of box $=25$ th percentile, bold line in box $=$ median, top of box $=75$ th percentile, top whisker $=95$ th percentile. $*$ Samples with values higher than twice the largest whisker are not illustrated. The number of stars $(*)$ indicates the number of samples not illustrated in the figures, within a specific polymer type. All values can be found in SM, Table A.1, where values excluded in the figure are highlighted in bold. 
Figure 4: Concentration of 15 metals $[\mu \mathrm{g} / \mathrm{g}]$ grouped based on the origin of the samples, illustrated with boxplots. At the boxplot: Lowest whisker = minimum concentration, bottom of box $=25$ th percentile, bold line in box $=$ median, top of box $=75$ th percentile, top whisker =95th percentile. "Waste" represents sample group 1, "rHHW": reprocessed plastic from HHW represents sample group 2, "rIW": reprocessed plastic from industrial waste represents sample group 3 and "virgin" represents sample group 4. * Samples with values higher than twice the largest whisker are not illustrated. The number of stars $(*)$ indicates the number of samples excluded with a specific origin. All values can be found in SM, Table A.1. 


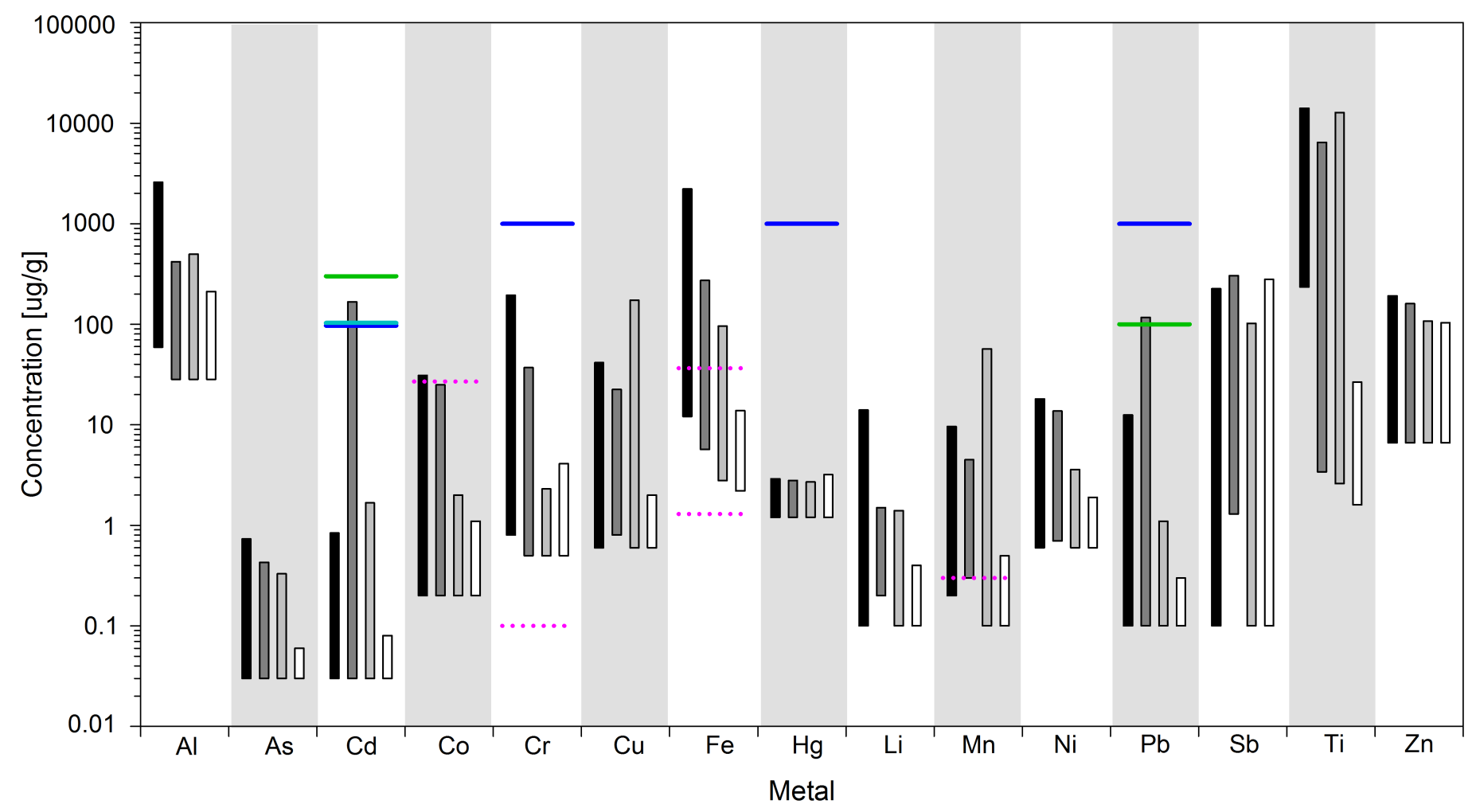

Plastic samples:

Waste

Legal limit values and literature data:

$\square$ Reprocessed from household waste

$\square$ Reprocessed from industrial waste

$\square$ Virgin

- EU limit for use (EU, 2006)

— EU limit for use in EE (EU, 2011b)

US limit for use in toys (Guney \& Zagury, 2012)

Concentrations in food packaging from literature

(Bach et al., 2012; 
Figure 5: Concentration ranges of all samples divided into groups of origin (sample group 14), EU limit values for use in electrical and electronic equipment (EE) and plastic in general, US limit values for use in toys and concentrations found in food plastic packaging from literature. The concentrations are presented on a logarithmic scale. 
Contamination in plastic recycling: Influence of metals on the quality of recycled plastic

\title{
Supplementary material
}

\author{
M. K. Eriksen", K. Pivnenko, T. F Astrup \\ Department of Environmental Engineering, Technical University of Denmark, DK-2800 Kgs. Lyngby, \\ Denmark
}

*) Corresponding author: Marie Kampmann Eriksen, maker@env.dtu.dk

\section{Table of Contents}

Appendix A Classification of metal containing substances used in plastic

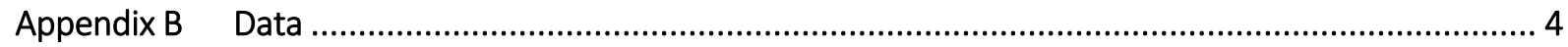

B.1 Metal concentration in all samples ……....................................................... 4

B.2 Geometric mean of all samples and based on origin ....................................... 7

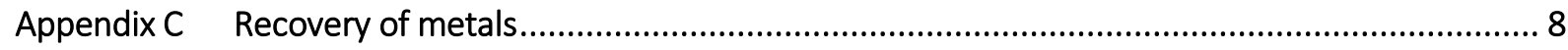

Appendix D Illustration of below detection limit ........................................................................... 9

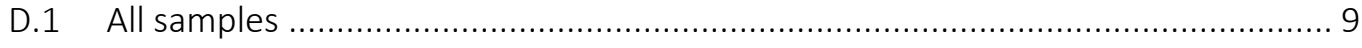

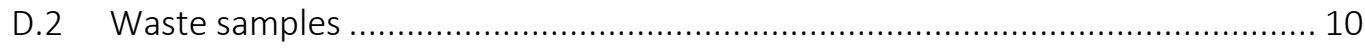

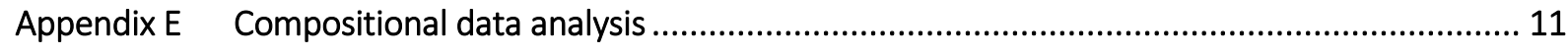

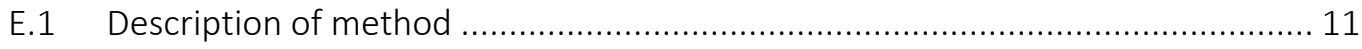

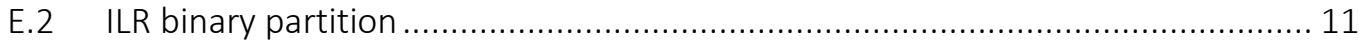

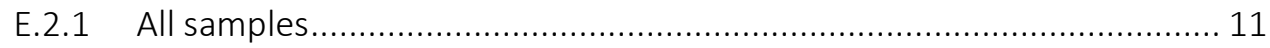

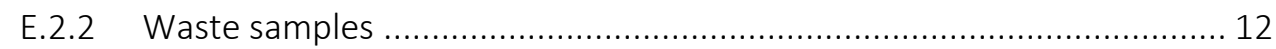

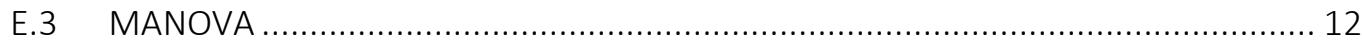

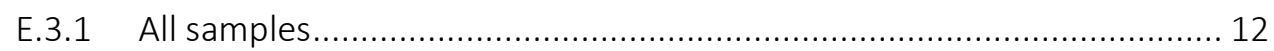

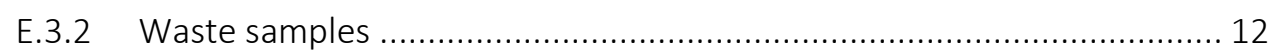

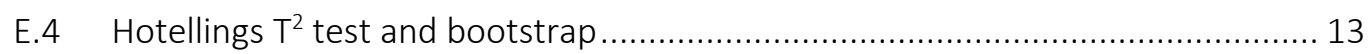

E.4.1 Grouping based on polymer (PET, PE, PP, PS) ................................ 13

E.4.2 Grouping based on origin (waste, rHHW, rIW and virgin) ...................... 15

E.4.3 Grouping based on washing - waste samples only ............................. 17

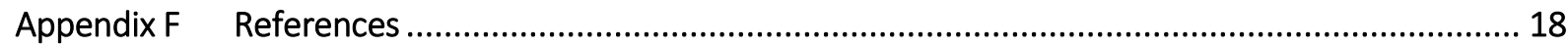




\section{Appendix A Classification of metal containing substances used in plastic}

Table A.1: Metallic compounds (name and CAS-no) known to be used in plastic (Hansen, 2013, Hahladakis et al., 2018)) listed according to classification (EC, 2008; ECHA, 2017)

\begin{tabular}{|c|c|c|c|}
\hline Carcinogenic & Mutagenic category 1-3 & Toxic for reproduction category 1-3 & Dangerous for the environment \\
\hline Category 1-3 & Category 1-3 & Category 1-3 & N, R52, R53 \\
\hline Arsenic oxides 1303-28-2 & Cadmium 7440-43-9 & Lead hydrogen arsenate 7784-40-9 & Arsenic 7440-38-2; \\
\hline Arsenic trioxides $1327-53-3$ & Cadmium chloride 10108-64-2 & Boric acid 10043-35-3 & Lead hydrogen arsenate 7784-40-9 \\
\hline Lead hydrogen arsenate 7784-40-9 & Cadmium fluoride 7790-79-6 & Cadmium 7440-43-9 & Arsine $7784-42-1$ \\
\hline Triethylarsenate 15606-95-8 & Cadmium oxide 1306-19-0 & Cadmium chloride 10108-64-2 & Cadmium cyanide 542-83-6 \\
\hline Cadmium 7440-43-9 & Cadmium sulphate 10124-36-4 & Cadmium fluoride 7790-79-6 & Cadmium fluoride 7790-79-6 \\
\hline Cadmium chloride 10108-64-2 & Cadmium sulphide 1306-23-6 & Cadmium oxide $1306-19-0$ & Cadmium formate $4464-23-7$ \\
\hline Cadmium cyanide 542-83-6 & Chromium (VI) trioxide 1333-82-0 & Cadmium sulphate 10124-36-4 & Cadmium iodide 7790-80-9 \\
\hline Cadmium fluoride 7790-79-6 & Potassium dichromate 7778-50-9 & Cadmium sulphide 1306-23-6 & Cadmium fluorosilica 17010- 21-8 \\
\hline Cadmium formate 4464-23-7 & Amonium dichromate 7789-09-5 & Chromium (VI) trioxide $1333-82-0$ & Cadmium oxide 1306-19-0 \\
\hline Cadmium iodide 7790-80-9 & Sodium dichromate 10588-01-9 & Potassium dichromate 7778-50-9 & Cadmium sulphate 10124-36-4 \\
\hline Cadmium fluorosilica 17010- 21-8 & Potassium chromate 7789-00-6 & Amonium dichromate 7789-09-5 & Cadmium sulphide 1306-23-6 \\
\hline Cadmium oxide 1306-19-0 & Sodium chromate 7775-11-3 & Sodium dichromate 10588-01-9 & Chromium (VI) trioxide 1333-82-0 \\
\hline Cadmium sulphate 10124-36-4 & Ammonium dichromate 7789-09-5 & $\begin{array}{l}\text { Lead chromate molybdate sulfate red } \\
\text { (C.I. Pigment red 104) 12656-85-8 }\end{array}$ & Potassium dichromate 7778-50-9 \\
\hline Cadmium sulphide 1306-23-6 & Chromyl dichloride 14977-61-8 & $\begin{array}{l}\text { Lead sulfochromate yellow (C.I. Pigment } \\
\text { Yellow 34) 1344-37-2 }\end{array}$ & Amonium dichromate 7789-09-5 \\
\hline Chromium (VI) trioxide 1333-82-0 & Cobalt di(acetate) 71-48-7 & Lead chromate 7758-97-6 & Sodium dichromate 10588-01-9 \\
\hline Potassium dichromate 7778-50-9 & Mercury dichloride 7487-94-7 & Sodium chromate $7775-11-3$ & Potassium chromate 7789-00-6 \\
\hline Amonium dichromate 7789-09-5 & & Ammonium dichromate 7789-09-5 & Calcium chromate 13765-19-0 \\
\hline Sodium dichromate 10588-01-9 & & Cobalt di(acetate) 71-48-7 & Strontium chromate 7789-06-2 \\
\hline Potassium chromate 7789-00-6 & & Lead di(acetate) 301-04-2 & Chromium III chromate 24613-89-6 \\
\hline Calcium chromate $13765-19-0$ & & Lead acetate 1335-32-6 & Lead chromate 7758-97-6 \\
\hline Strontium chromate 7789-06-2 & & trilead bis(orthophosphate) 7446-27-7 & Sodium chromate 7775-11-3 \\
\hline Chromium III chromate 24613-89-6 & & Lead chromate 7758-97-6 & Ammonium dichromate 7789-09-5 \\
\hline $\begin{array}{l}\text { Lead chromate molybdate sulfate red } \\
\text { (C.I. Pigment red 104) 12656-85-8 }\end{array}$ & & Lead diazide 13424-46-9 & Chromyl dichloride 14977-61-8 \\
\hline
\end{tabular}


Table A.1 (continued): Metallic compounds (name and CAS-no) known to be used in plastic (Hansen, 2013; Hahladakis et al., 2018) listed according to classification (EC, 2008; ECHA, 2017)

\begin{tabular}{|c|c|c|c|}
\hline Carcinogenic & Mutagenic category 1-3 & Toxic for reproduction category 1-3 & Dangerous for the environment \\
\hline Category 1-3 & Category 1-3 & Category 1-3 & N, R52, R53 \\
\hline $\begin{array}{l}\text { Lead sulfochromate yellow (C.I. Pigment } \\
\text { Yellow 34) 1344-37-2 }\end{array}$ & & Lead stypnate 15245-44-0 & Cobalt di(acetate) 71-48-7 \\
\hline Lead chromate 7758-97-6 & & Lead(II) methanesulphonate 17570-76-2 & Lead di(acetate) 301-04-2 \\
\hline Sodium chromate 7775-11-3 & & Lead hexafluorosilicate 25808-74-6 & Lead acetate 1335-32-6 \\
\hline Ammonium dichromate 7789-09-5 & & Other lead compounds & trilead bis(orthophosphate) 7446-27-7 \\
\hline Chromyl dichloride 14977-61-8 & & Mercury 7439-97-6 & Lead chromate 7758-97-6 \\
\hline Cobalt di(acetate) 71-48-7 & & Mercury dichloride 7487-94-7 & Lead diazide 13424-46-9 \\
\hline Lead acetate 1335-32-6 & & & Lead stypnate 15245-44-0 \\
\hline Lead chromate 7758-97-6 & & & Lead(II) methanesulphonate 17570-76-2 \\
\hline (Titanium dioxide 13463-67-7)* & & & Lead hexafluorosilicate 25808-74-6 \\
\hline \multirow[t]{15}{*}{ Antimony trioxide $1309-64-4$} & & & Other lead compounds \\
\hline & & & Other cadmium compounds \\
\hline & & & Phenylmercury nitrate 55-68-5 \\
\hline & & & Phenylmercury acetate 62-38-4 \\
\hline & & & Phenylmercury hydroxide 100-57-2 \\
\hline & & & 2-methoxyethylmercury chloride 123-88-6 \\
\hline & & & Dimethylmercury 593-74-8 \\
\hline & & & Mercury fulminates 628-86-4 \\
\hline & & & Mercuric oxycyanide 1335-31-5 \\
\hline & & & Mercury 7439-97-6 \\
\hline & & & Mercury dichloride 7487-94-7 \\
\hline & & & Phenylmercury nitrate 8003-05-2 \\
\hline & & & Dimercury dichloride 10112-91-1 \\
\hline & & & Other antimony compounds \\
\hline & & & Other mercury compounds \\
\hline
\end{tabular}

\footnotetext{
* Proposed to be classified as suspected of causing cancer when inhaled (ECHA, 2017)
} 


\section{Appendix B Data}

\section{B.1 Metal concentration in all samples}

Table B.1 Concentration of all metals in all samples. Values not depicted in Figure 2 and 3 in the main paper are underlined and highlighted in bold. The value for each sample is the geometric mean of the triplicates. rHHW: reprocessed plastic from household waste, rIW: reprocessed plastic from industrial waste.

\begin{tabular}{|c|c|c|c|c|c|c|c|c|c|c|c|c|c|c|c|c|c|c|}
\hline \# & Sample group & Type & Polymer & $\mathrm{Al}$ & As & $\mathrm{Cd}$ & Co & $\mathrm{Cr}$ & $\mathrm{Cu}$ & $\mathrm{Fe}$ & $\mathrm{Hg}$ & Li & $\mathrm{Mn}$ & $\mathrm{Ni}$ & $\mathrm{Pb}$ & $\mathrm{Sb}$ & $\mathrm{Ti}$ & $\mathrm{Zn}$ \\
\hline 1 & 5 & virgin & PET & 28.4 & 0.03 & 0.03 & 0.21 & 0.48 & 0.65 & 7.15 & 1.17 & 0.14 & 0.27 & 1.30 & 0.20 & 279 & 25.1 & 6.60 \\
\hline 2 & 4 & rIW & PET & 88.5 & 0.03 & 0.03 & 0.19 & 0.48 & 0.65 & 9.73 & 1.17 & 0.16 & $\underline{56.7}$ & 0.58 & 0.15 & 0.38 & 19.2 & 6.60 \\
\hline 3 & 4 & rIW & PET & 53.7 & 0.03 & 0.03 & 0.38 & 0.48 & 0.75 & 4.05 & 1.38 & 0.14 & 3.68 & 0.58 & 0.17 & 102 & 1620 & 6.60 \\
\hline 4 & 3 & $\mathrm{rHHW}$ & PET & 45.1 & 0.03 & 0.03 & 3.13 & 2.39 & 0.75 & 5.69 & 1.17 & 0.18 & 0.30 & 0.67 & 0.13 & 271 & 6.43 & 6.60 \\
\hline 5 & 3 & $\mathrm{rHHW}$ & PET & 28.4 & 0.03 & 0.03 & 10.74 & 0.48 & 1.07 & 17.5 & 1.17 & 0.21 & 0.36 & 6.31 & 0.13 & 304 & 3.39 & 6.60 \\
\hline 6 & 2.1 & waste & PET & 440 & 0.14 & 0.03 & 8.10 & 10.5 & 41.0 & 362 & 1.17 & 0.54 & 5.43 & 3.26 & 0.65 & 226 & 1500 & 21.0 \\
\hline 7 & 2.2 & waste & PET & 516 & 0.09 & 0.03 & 6.19 & 8.46 & 9.96 & 401 & 1.43 & 0.24 & 3.09 & 0.90 & 0.23 & 224 & 1340 & 9.91 \\
\hline 8 & 1.1 & waste & PET & 278 & 0.05 & 0.03 & 3.38 & 11.4 & 2.71 & 364 & 1.17 & 0.25 & 5.01 & 1.57 & 0.40 & 181 & 322 & 6.60 \\
\hline 9 & 1.3 & waste & PET & 160 & 0.04 & 0.03 & 1.09 & 5.55 & 1.89 & 297 & 1.73 & 0.14 & 2.97 & 0.70 & 0.28 & 211 & 599 & 6.60 \\
\hline 10 & 1.2 & waste & PET & 160 & 0.06 & 0.03 & 4.79 & 5.40 & 1.92 & 194 & 1.45 & 0.19 & 2.23 & 1.08 & 0.53 & 226 & 753 & 8.32 \\
\hline 11 & 1.4 & waste & PET & 625 & 0.04 & 0.03 & 2.65 & 1.39 & 3.08 & 86.5 & 1.17 & 0.53 & 1.10 & 1.10 & 0.38 & 168 & 877 & 8.44 \\
\hline 12 & 1.1 & waste & PET & 802 & 0.48 & 0.03 & 7.75 & 10.7 & 7.22 & 426 & 1.17 & 0.56 & 6.68 & 1.86 & 1.00 & 169 & 2560 & 6.60 \\
\hline 13 & 1.3 & waste & PET & 266 & 0.20 & 0.03 & 0.82 & 3.76 & 1.81 & 150 & 1.17 & 0.56 & 2.52 & 0.57 & 0.46 & 129 & 6670 & 6.60 \\
\hline 14 & 1.2 & waste & PET & 182 & 0.11 & 0.03 & 6.12 & 3.31 & 1.35 & 144 & 1.17 & 0.14 & 1.57 & 0.76 & 0.25 & 112 & 1810 & 9.19 \\
\hline 15 & 1.4 & waste & PET & 68.1 & 0.04 & 0.04 & 0.58 & 0.85 & 0.65 & 23.2 & 1.41 & 0.14 & 0.55 & 0.58 & 0.28 & 20.5 & 1420 & 6.60 \\
\hline 16 & 5 & virgin & PE & 32.5 & 0.03 & 0.03 & 0.33 & 0.86 & 0.65 & 3.61 & 1.17 & 0.14 & 0.27 & 0.82 & 0.13 & 0.09 & 6.87 & 6.60 \\
\hline 17 & 5 & virgin & $\mathrm{PE}$ & 85.4 & 0.03 & 0.03 & 0.19 & 0.48 & 0.65 & 2.24 & 1.17 & 0.14 & 0.13 & 0.58 & 0.13 & 0.09 & 6.75 & 26.5 \\
\hline 18 & 5 & virgin & PE & 133 & 0.03 & 0.03 & 0.19 & 0.56 & 0.65 & 3.14 & 1.17 & 0.17 & 0.13 & 0.69 & 0.15 & 0.09 & 8.58 & 43.6 \\
\hline 19 & 5 & virgin & PE & 108 & 0.03 & 0.03 & 0.19 & 0.48 & 0.65 & 2.24 & 1.17 & 0.14 & 0.13 & 0.58 & 0.17 & 0.09 & 6.45 & 6.60 \\
\hline 20 & 4 & rIW & PE & 91.3 & 0.12 & 0.18 & 0.19 & 1.83 & 2.76 & 17.7 & 1.17 & 0.37 & 0.29 & 0.58 & 0.53 & 0.61 & 1780 & 20.6 \\
\hline 21 & 4 & rIW & PE & 147 & 0.03 & $\underline{1.64}$ & 0.19 & 1.44 & 4.89 & 93.1 & 1.46 & 0.17 & 1.70 & 0.58 & 1.04 & 1.10 & 829 & 22.6 \\
\hline 22 & 3 & rHHW & PE & 195 & 0.10 & $\underline{4.50}$ & 0.67 & 2.11 & 4.50 & 43.7 & 1.17 & 0.36 & 0.70 & 1.62 & 3.20 & 2.54 & 2460 & 150 \\
\hline 23 & 3 & $\mathrm{rHHW}$ & $P E$ & 272 & 0.08 & $\underline{167}$ & $\underline{24.8}$ & $\underline{36.5}$ & 19.3 & 268 & 1.61 & 0.34 & 4.23 & $\underline{13.0}$ & $\underline{117}$ & 16.4 & 1130 & 92.1 \\
\hline
\end{tabular}


Table B.1 continued

\begin{tabular}{|c|c|c|c|c|c|c|c|c|c|c|c|c|c|c|c|c|c|c|}
\hline$\#$ & Sample group & Type & Polymer & $\mathrm{Al}$ & As & $\mathrm{Cd}$ & Co & $\mathrm{Cr}$ & $\mathrm{Cu}$ & $\mathrm{Fe}$ & $\mathrm{Hg}$ & $\mathrm{Li}$ & $\mathrm{Mn}$ & $\mathrm{Ni}$ & $\mathrm{Pb}$ & $\mathrm{Sb}$ & $\mathrm{Ti}$ & $\mathrm{Zn}$ \\
\hline 24 & 3 & $\mathrm{rHHW}$ & PE & 80.8 & 0.03 & $\underline{1.31}$ & 0.19 & 3.35 & 20.4 & 73.5 & 1.17 & 0.20 & 1.64 & 1.07 & 7.07 & 1.33 & 1030 & 24.9 \\
\hline 25 & 3 & $\mathrm{rHHW}$ & $P E$ & 224 & 0.04 & 0.24 & 0.19 & 1.79 & 5.81 & 195 & 1.42 & 0.26 & 3.60 & 1.79 & 2.64 & 1.66 & 971 & 154 \\
\hline 26 & 2.1 & waste & PE & 444 & 0.19 & 0.04 & 1.33 & 4.87 & 11.3 & 208 & 1.17 & 0.25 & 3.01 & 1.33 & 0.38 & 3.66 & 4070 & 57.3 \\
\hline 27 & 2.2 & waste & PE & 290 & 0.24 & 0.03 & $\underline{30.7}$ & 2.68 & 16.2 & 50.4 & 1.17 & 0.30 & 1.05 & $\underline{17.5}$ & 0.18 & 7.51 & 5330 & 85.7 \\
\hline 28 & 1.1 & waste & PE & 183 & 0.10 & 0.03 & 2.06 & 6.03 & 12.8 & 187 & 1.17 & 0.28 & 2.83 & 1.47 & 0.21 & 0.23 & 1770 & 7.86 \\
\hline 29 & 1.3 & waste & PE & 354 & 0.12 & 0.03 & 1.19 & 8.07 & 14.3 & 304 & 1.46 & 0.42 & 4.96 & 2.84 & 0.63 & 0.26 & 2300 & 32.1 \\
\hline 30 & 1.2 & waste & $P E$ & 160 & 0.09 & 0.03 & 4.51 & 1.64 & 6.75 & 63.2 & 1.65 & 0.29 & 1.42 & 2.80 & 0.15 & 0.39 & 4110 & 15.9 \\
\hline 31 & 1.4 & waste & PE & 178 & 0.04 & 0.03 & 0.31 & 1.34 & 1.41 & 71.3 & 1.17 & 0.14 & 1.66 & 0.58 & 0.30 & 0.27 & 3150 & 6.60 \\
\hline 32 & 1.1 & waste & $P E$ & 241 & 0.03 & 0.03 & 0.30 & 1.02 & 0.75 & 60.3 & 1.17 & 0.19 & 1.46 & 0.58 & 0.19 & 1.09 & 2140 & 6.60 \\
\hline 33 & 1.3 & waste & PE & 237 & 0.04 & 0.03 & 2.50 & 7.57 & 3.10 & 149 & 1.17 & 0.14 & 2.46 & 0.58 & 0.57 & 0.50 & 1720 & 9.35 \\
\hline 34 & 1.2 & waste & PE & 59.3 & 0.03 & 0.03 & 4.70 & 2.67 & 0.65 & 17.9 & 1.45 & 0.14 & 0.31 & 2.27 & 0.13 & 0.14 & 2370 & 8.21 \\
\hline 35 & 1.4 & waste & $P E$ & 273 & 0.07 & 0.03 & 0.38 & 1.44 & 0.83 & 12.1 & 1.17 & 0.14 & 0.18 & 0.58 & 0.28 & 1.84 & 13800 & 16.1 \\
\hline 36 & 5 & virgin & PE & 28.4 & 0.03 & 0.03 & 0.19 & 0.48 & 0.65 & 2.24 & 1.36 & 0.14 & 0.13 & 0.58 & 0.13 & 0.09 & 3.80 & 6.60 \\
\hline 37 & 5 & virgin & $P E$ & 28.4 & 0.03 & 0.03 & 0.19 & 0.48 & 0.65 & 2.70 & 1.17 & 0.20 & 0.13 & 0.58 & 0.13 & 0.09 & 1.56 & 6.60 \\
\hline 38 & 5 & virgin & PE & 28.4 & 0.03 & 0.03 & 0.19 & 0.66 & 0.65 & 7.30 & 1.17 & 0.30 & 0.13 & 0.75 & 0.13 & 0.09 & 2.57 & 6.60 \\
\hline 39 & 4 & rIW & PE & 28.4 & 0.03 & 0.03 & 0.19 & 0.48 & 0.83 & 2.80 & 1.17 & 0.14 & 0.13 & 0.58 & 0.13 & 0.09 & 11.2 & 6.60 \\
\hline 40 & 4 & rIW & $P E$ & 12 & 0.06 & 0.03 & 0.19 & 0.48 & 0.76 & 5.37 & 1.17 & 0.30 & 0.15 & 0.67 & 0.13 & 0.09 & 9020 & 6.60 \\
\hline 41 & 4 & rIW & PE & 213 & 0.18 & 0.03 & 0.19 & 0.48 & 0.65 & 23.9 & 1.17 & 0.45 & 2.20 & 0.58 & 0.73 & 0.09 & 10300 & 26.7 \\
\hline 42 & 4 & rIW & $P E$ & 122 & 0.03 & 0.03 & 0.19 & 0.48 & 0.65 & 12.1 & 1.17 & 0.14 & 0.24 & 0.58 & 0.17 & 0.09 & 17.4 & 8.23 \\
\hline 43 & 4 & rIW & PE & 470 & 0.05 & 0.03 & 0.38 & 0.88 & 2.52 & 34.7 & 1.17 & 0.15 & 1.03 & 0.81 & 0.37 & 0.17 & 492 & 27.5 \\
\hline 44 & 4 & rIW & $P E$ & 145 & 0.03 & 0.03 & 0.19 & 0.73 & 0.84 & 74.6 & 1.17 & 0.23 & 2.39 & 0.89 & 0.44 & 0.31 & 44.1 & 59.2 \\
\hline 45 & 3 & $\mathrm{rHHW}$ & $P E$ & 345 & 0.39 & 0.43 & 0.49 & 4.83 & 15.9 & 110 & 1.17 & 0.21 & 4.25 & 1.38 & 12.3 & 36.3 & 6460 & 97.9 \\
\hline 46 & 5 & virgin & $P E$ & 75.5 & 0.03 & 0.03 & 0.54 & 0.55 & 0.98 & 6.68 & 1.17 & 0.14 & 0.13 & 1.33 & 0.13 & 0.09 & 3.14 & 67.1 \\
\hline 47 & 4 & rIW & $P E$ & 118 & 0.03 & 0.03 & 0.19 & 1.41 & 0.65 & 45.6 & 1.17 & 0.27 & 1.87 & 0.58 & 0.17 & 0.17 & 28.3 & 6.60 \\
\hline 48 & 4 & rIW & $P E$ & 117 & 0.03 & 0.03 & 0.19 & 0.58 & 0.65 & 19.8 & 1.17 & 0.36 & 0.46 & 0.97 & 0.13 & 0.09 & 19.3 & 101 \\
\hline 49 & 4 & rIW & PE & 28.4 & 0.03 & 0.03 & 0.19 & 0.48 & 0.65 & 7.68 & 1.17 & 0.17 & 0.13 & 0.58 & 0.13 & 0.09 & 12.5 & 6.60 \\
\hline
\end{tabular}


Table B.1 Continued

\begin{tabular}{|c|c|c|c|c|c|c|c|c|c|c|c|c|c|c|c|c|c|c|}
\hline$\#$ & Sample group & Type & Polymer & $\mathrm{Al}$ & As & $\mathrm{Cd}$ & Co & $\mathrm{Cr}$ & $\mathrm{Cu}$ & $\mathrm{Fe}$ & $\mathrm{Hg}$ & $\mathrm{Li}$ & $\mathrm{Mn}$ & $\mathrm{Ni}$ & $\mathrm{Pb}$ & $\mathrm{Sb}$ & $\mathrm{Ti}$ & $\mathrm{Zn}$ \\
\hline 50 & 5 & virgin & $\mathrm{PP}$ & 183 & 0.03 & 0.05 & 0.94 & 0.48 & 1.36 & 11.6 & 2.01 & 0.14 & 0.38 & 1.03 & 0.15 & 0.18 & 5.52 & 10.5 \\
\hline 51 & 5 & virgin & $\mathrm{PP}$ & 58.1 & 0.03 & 0.03 & 0.19 & 0.48 & 0.65 & 5.99 & 1.17 & 0.14 & 0.16 & 0.58 & 0.13 & 0.09 & 4.39 & 6.60 \\
\hline 52 & 5 & virgin & $P P$ & 59.9 & 0.03 & 0.03 & 0.29 & 0.48 & 0.65 & 2.82 & 1.17 & 0.14 & 0.13 & 0.58 & 0.13 & 0.09 & 2.57 & 6.60 \\
\hline 53 & 5 & virgin & $\mathrm{PP}$ & 46.7 & 0.03 & 0.03 & 0.19 & 3.65 & 0.65 & 5.31 & 1.17 & 0.22 & 0.13 & 0.94 & 0.13 & 0.09 & 6.35 & 6.60 \\
\hline 54 & 4 & rIW & PP & 28.4 & 0.05 & 0.03 & 1.84 & 0.48 & 2.20 & 3.72 & 1.17 & 0.14 & 0.13 & 3.02 & 0.13 & 0.09 & 3.58 & 6.60 \\
\hline 55 & 4 & rIW & $P P$ & 443 & 0.32 & 0.03 & 1.24 & 0.91 & 173 & 36.0 & 1.45 & 0.17 & 3.68 & 1.03 & 0.40 & 1.08 & 12800 & 69.7 \\
\hline 56 & 4 & rIW & $\mathrm{PP}$ & 38.1 & 0.03 & 0.03 & 0.43 & 0.48 & 0.96 & 3.15 & 1.17 & 0.14 & 0.13 & 0.69 & 0.13 & 0.09 & 6.00 & 6.60 \\
\hline 57 & 3 & rHHW & $P P$ & 390 & 0.15 & $\underline{5.03}$ & 1.12 & 2.57 & 21.7 & 226 & 1.64 & 0.67 & 2.07 & 0.68 & 12.44 & 15.8 & 3660 & 32.8 \\
\hline 58 & 3 & rHHW & $P P$ & 287 & 0.06 & 5.81 & 1.24 & 7.37 & 10.1 & 173 & 1.17 & 1.29 & 2.35 & 1.17 & 18.40 & 33.1 & 1970 & 12.2 \\
\hline 59 & 3 & rHHW & $\mathrm{PP}$ & 207 & 0.10 & 15.9 & 1.54 & 14.3 & 11.4 & 220 & 1.17 & 0.28 & 3.33 & 1.85 & 42.8 & 5.84 & 1740 & 34.5 \\
\hline 60 & 2.1 & waste & $P P$ & 839 & 0.45 & 0.24 & 1.70 & 24.8 & 18.0 & 1060 & 1.17 & 0.68 & 9.12 & 7.68 & 7.98 & 3.73 & 1710 & 35.6 \\
\hline 61 & 2.2 & waste & $\mathrm{PP}$ & 2522 & 0.42 & 0.20 & 6.23 & 30.0 & 19.2 & 723 & 1.41 & 0.24 & 4.28 & 11.0 & 6.23 & 3.11 & 2070 & 11.0 \\
\hline 62 & 1.1 & waste & $P P$ & 627 & 0.28 & 0.03 & 1.49 & 8.02 & 16.9 & 267 & 1.17 & 0.40 & 4.58 & 2.61 & 0.92 & 0.43 & 3880 & 18.6 \\
\hline 63 & 1.3 & waste & $P P$ & 143 & 0.03 & 0.23 & 0.44 & 12.9 & 3.05 & 85.4 & 1.36 & 0.67 & 1.20 & 0.89 & 12.42 & 0.35 & 235 & 14.7 \\
\hline 64 & 1.2 & waste & $P P$ & 86.9 & 0.09 & 0.03 & 0.22 & 0.99 & 2.48 & 59.8 & 1.17 & 0.32 & 0.80 & 0.58 & 0.13 & 0.11 & 2760 & 11.1 \\
\hline 65 & 1.4 & waste & $P P$ & 145 & 0.07 & 0.18 & 1.12 & 2.67 & 14.9 & 109 & 1.43 & 0.75 & 1.38 & 0.79 & 0.82 & 2.29 & 1320 & 15.0 \\
\hline 66 & 1.1 & waste & PP & 199 & 0.68 & 0.03 & 4.71 & 2.13 & 10.9 & 114 & 1.17 & 0.14 & 3.02 & 2.63 & 0.58 & 0.09 & 7000 & 8.24 \\
\hline 67 & 1.3 & waste & PP & 210 & 0.33 & 0.11 & 1.13 & 6.83 & 32.50 & 1520 & 1.17 & 0.32 & 8.22 & 3.52 & 2.11 & 0.42 & 3810 & 26.1 \\
\hline 68 & 1.2 & waste & $P P$ & 182 & 0.43 & 0.03 & 0.77 & 0.76 & 0.65 & 55.0 & 1.54 & 0.14 & 0.93 & 0.99 & 0.23 & 1.31 & 7980 & 6.60 \\
\hline 69 & 1.4 & waste & PP & 297 & 0.42 & 0.03 & 4.21 & 18.0 & 27.6 & 2210 & 1.17 & 0.14 & 7.99 & 5.95 & 0.23 & 0.14 & 4000 & 6.60 \\
\hline 70 & 5 & virgin & PS & 28.4 & 0.03 & 0.03 & 0.34 & 0.48 & 0.65 & 2.24 & 1.17 & 0.14 & 0.13 & 0.58 & 0.19 & 0.27 & 2.57 & 85.6 \\
\hline 71 & 5 & virgin & PS & 28.4 & 0.03 & 0.03 & 0.19 & 1.90 & 0.65 & 2.24 & 1.17 & 0.14 & 0.13 & 0.58 & 0.23 & 0.09 & 2.57 & 63.2 \\
\hline 72 & 5 & virgin & PS & 28.4 & 0.03 & 0.03 & 0.52 & 0.48 & 0.65 & 2.24 & 1.17 & 0.16 & 0.13 & 0.58 & 0.13 & 0.09 & 2.57 & 97.1 \\
\hline 73 & 5 & virgin & PS & 28.4 & 0.03 & 0.03 & 0.91 & 0.48 & 0.75 & 8.06 & 1.17 & 0.17 & 0.24 & 0.58 & 0.21 & 0.09 & 5.90 & 53.7 \\
\hline 74 & 4 & rIW & PS & 360 & 0.03 & 0.03 & 0.83 & 0.66 & 1.93 & 7.13 & 1.17 & 0.73 & 0.23 & 1.06 & 0.13 & 0.09 & 3500 & 55.2 \\
\hline 75 & 4 & rIW & PS & 43.6 & 0.03 & 0.03 & 0.25 & 0.66 & 0.65 & 30.4 & 1.17 & 0.23 & 0.36 & 0.84 & 0.33 & 0.09 & 2.57 & 82.4 \\
\hline
\end{tabular}


Table B.1 Continued

\begin{tabular}{|c|c|c|c|c|c|c|c|c|c|c|c|c|c|c|c|c|c|c|}
\hline$\#$ & Sample group & Type & Polymer & $\mathrm{Al}$ & As & $\mathrm{Cd}$ & Co & $\mathrm{Cr}$ & $\mathrm{Cu}$ & $\mathrm{Fe}$ & $\mathrm{Hg}$ & $\mathrm{Li}$ & $\mathrm{Mn}$ & $\mathrm{Ni}$ & $\mathrm{Pb}$ & $\mathrm{Sb}$ & $\mathrm{Ti}$ & $\mathrm{Zn}$ \\
\hline 76 & 4 & rIW & PS & 43.5 & 0.04 & 0.03 & 0.19 & 0.48 & 0.65 & 24.2 & 1.38 & 1.32 & 0.13 & 0.58 & 0.22 & 0.12 & 1500 & 79.7 \\
\hline 77 & 2.1 & waste & PS & 2476 & 0.25 & 0.30 & 1.00 & 4.18 & 4.28 & 244 & 1.17 & 5.42 & 5.43 & 1.69 & 1.27 & 6.94 & 2290 & 91.6 \\
\hline 78 & 2.2 & waste & PS & 270 & 0.27 & 0.39 & 1.24 & 1.45 & 3.41 & 129 & 1.17 & 7.85 & 1.79 & 1.22 & 1.42 & 15.0 & 2880 & 76.2 \\
\hline 79 & 1.1 & waste & PS & 1472 & 0.28 & 0.03 & 1.13 & 12.8 & 8.81 & 339 & 1.17 & $\underline{13.9}$ & 9.41 & 4.09 & 1.07 & 0.37 & 1820 & 87.5 \\
\hline 80 & 1.3 & waste & PS & 248 & 0.05 & 0.16 & 4.85 & 19.2 & 3.88 & 206 & 1.17 & 2.41 & 2.31 & 1.01 & 9.59 & 12.9 & 1650 & 106 \\
\hline 81 & 1.2 & waste & PS & 370 & 0.14 & 0.81 & 0.22 & 1.03 & 2.88 & 55.3 & 1.53 & 5.30 & 1.34 & 0.58 & 1.00 & 1.19 & 6870 & 118 \\
\hline 82 & 1.4 & waste & PS & 234 & 0.13 & 0.05 & 24.8 & $\underline{193}$ & 4.03 & 356 & 1.37 & 1.01 & 2.21 & 4.92 & 0.57 & 2.03 & 964 & 186 \\
\hline
\end{tabular}

\section{B.2 Geometric mean of all samples and based on origin}

Table B.2: Geometric mean of all samples, waste samples, reprocessed samples from household waste (rHHW), reprocessed samples from industrial waste (rIW) and virgin samples for all metals. All values are given in $\mu \mathrm{g} / \mathrm{g}$.

\begin{tabular}{l|c|c|c|c|c|c|c|c|c|c|c|c|c|c|c|c|c|c|}
\hline Geometric mean of: & $\mathrm{Al}$ & $\mathrm{As}$ & $\mathrm{Cd}$ & $\mathrm{Co}$ & $\mathrm{Cr}$ & $\mathrm{Cu}$ & $\mathrm{Fe}$ & $\mathrm{Hg}$ & $\mathrm{Li}$ & $\mathrm{Mn}$ & $\mathrm{Ni}$ & $\mathrm{Pb}$ & $\mathrm{Sb}$ & $\mathrm{Ti}$ & $\mathrm{Zn}$ \\
\hline All samples & 145 & 0.06 & 0.06 & 0.79 & 1.92 & 2.49 & 39.35 & 1.25 & 0.29 & 0.94 & 1.14 & 0.47 & 1.23 & 281 & 19.1 \\
\hline Waste samples & 293 & 0.12 & 0.05 & 1.90 & 4.76 & 4.68 & 161 & 1.27 & 0.42 & 2.25 & 1.58 & 0.59 & 3.72 & 2197 & 17.4 \\
rHHW samples & 158 & 0.07 & 1.43 & 1.35 & 3.84 & 7.10 & 83.1 & 1.27 & 0.32 & 1.63 & 1.80 & 5.18 & 15.6 & 588 & 35.4 \\
rlW samples & 96.6 & 0.04 & 0.04 & 0.29 & 0.66 & 1.35 & 14.5 & 1.22 & 0.24 & 0.64 & 0.75 & 0.23 & 0.24 & 156 & 18.7 \\
Virgin samples & 48.0 & 0.03 & 0.03 & 0.28 & 0.63 & 0.70 & 3.90 & 1.22 & 0.16 & 0.16 & 0.71 & 0.15 & 0.16 & 4.49 & 16.6 \\
\hline
\end{tabular}




\section{Appendix C Recovery of metals}

Table C.1 Recovery [\%] of 10 metals, found using PerkinElmer Quality control, 23 elements.

\begin{tabular}{|c|c|c|c|c|c|c|c|c|c|c|c|c|c|c|c|}
\hline & $\mathrm{Al}$ & As & $\mathrm{Cd}$ & Co & $\mathrm{Cr}$ & $\mathrm{Cu}$ & $\mathrm{Fe}$ & $\mathrm{Hg}$ & $\mathrm{Li}$ & $\mathrm{Mn}$ & $\mathrm{Ni}$ & $\mathrm{Pb}$ & $\mathrm{Sb}$ & $\mathrm{Ti}$ & $\mathrm{Zn}$ \\
\hline Recovery [\%] & & 85.4 & 87.3 & 133 & 117 & & & 93.0 & 83.3 & 91.3 & 120 & 94.1 & 96.0 & & \\
\hline
\end{tabular}




\section{Appendix D Illustration of below detection limit}

\section{D.1 All samples}

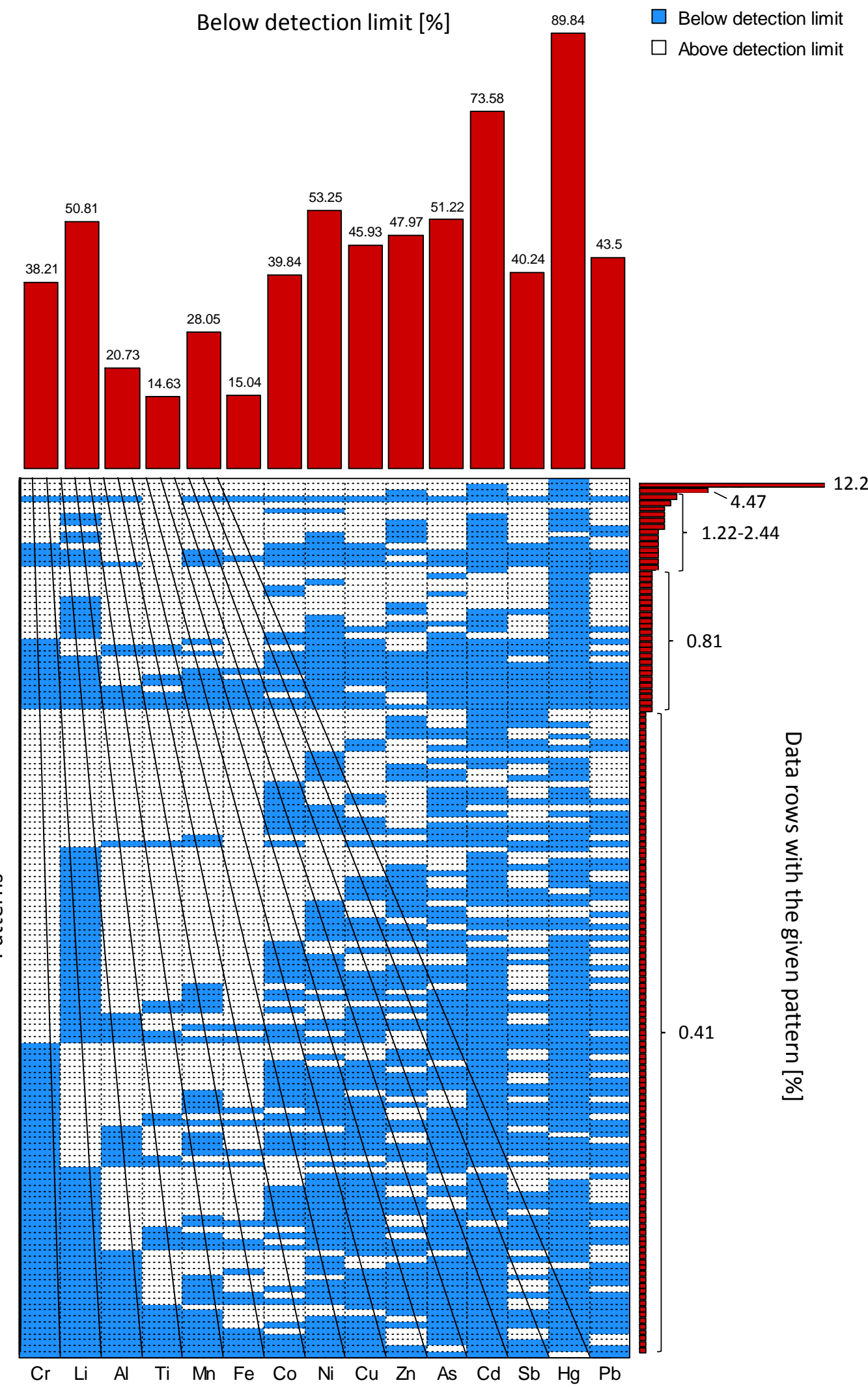

Figure D.1 Illustration of frequency of patterns in the dataset [\%] (right axis) and the frequency of values below the detection limit [\%] (top axis) for all samples. 


\section{D.2 Waste samples}

Below detection limit [\%]
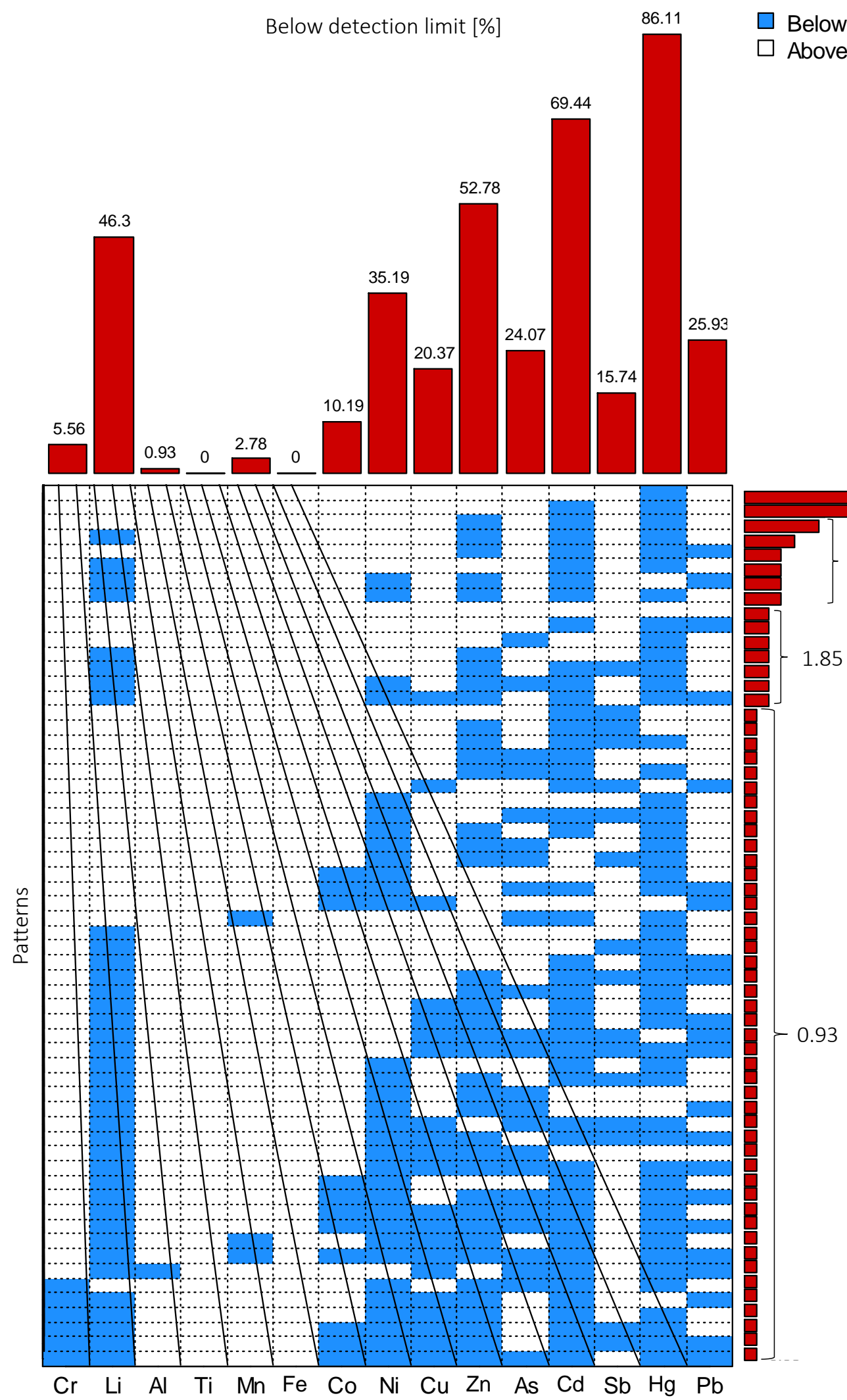

Figure D.2 Illustration of frequency of patterns in the dataset [\%] (right axis) and the frequency of values below the detection limit [\%] (top axis) for waste samples. 


\section{Appendix E Compositional data analysis}

\section{E.1 Description of method}

Analytical data were transformed using isometric log-ratio (ilr) transformation (Egozcue et al., 2003), as recommended by Edjabout et al. (2017). Again, this was applied twice: once for the total dataset and once for the waste sample dataset only. The transformation was done using freely available software for compositional data analysis (CoDaPack, version 2.02.04, retrieved from http://www.compositionaldata.com). To assess potential statistical differences between groups, the following statistical operations were performed on the transformed data (Edjabou, 2016):

- Multivariate analysis of variance (MANOVA): MANOVA was performed to evaluate if there was a significant difference in metal concentrations between groups (e.g. between different polymer types or origins). The level of significance, $\alpha 0$, was 0.05 , as recommended by Edjabou et al. (2016).

- Hotelling's T2 test: if the MANOVA showed a significant difference between groups (e.g. samples of different origins), this test was used to evaluate which of the groups (e.g. waste, reprocessed from HHW, reprocessed from IW and virgin samples) were responsible for the significant difference. As an example, metal concentrations in waste samples and virgin samples (sample group 1 compared to 4) could be significantly different, even though the difference in metal concentrations between reprocessed samples from industry and virgin samples was insignificant (sample group 3 compared to 4 ). The level of significance, $\alpha 1$, was $\alpha 1=\alpha 0 /(G *(G-1) / 2)$, where $G$ is the number of groups ( $G=4$ for both polymer type and origin for the total dataset [ $\alpha 1=0.0083]$. $G=2$ for washing, for the waste dataset [ $\alpha 1=0.05]$ ).

- Bootstrap: this analysis assesses which variables (metals) are responsible for a significant difference between two groups. As recommended by Edjabou et al. (2016), the level of significance, $\alpha 2$, was $\alpha 2=\alpha 1 / P$, where $P$ is the number of metals with frequency of detection $(F O D)>50 \%$ for all observations ( $P=10$ for the total dataset $[\alpha 2=0.00083$ ]. $P=12$ for the waste dataset $[\alpha 2=0.0041]$, see Appendix $D$ for details).

\section{E.2 ILR binary partition}

\section{E.2.1 All samples}

\section{$\mathrm{Cr} \mathrm{Al} \mathrm{Ti} \mathrm{Mn} \mathrm{Fe} \mathrm{Co} \mathrm{Cu} \mathrm{Zn} \mathrm{Sb} \mathrm{Pb} \mathrm{Ir}$}

$\begin{array}{rrrrrrrrrrr}1 & 1 & 1 & 1 & 1 & -1 & -1 & -1 & -1 & -1 & 1 \\ 1 & 1 & 1 & -1 & -1 & 0 & 0 & 0 & 0 & 0 & 2 \\ 1 & 1 & -1 & 0 & 0 & 0 & 0 & 0 & 0 & 0 & 3 \\ 1 & -1 & 0 & 0 & 0 & 0 & 0 & 0 & 0 & 0 & 4 \\ 0 & 0 & 0 & 1 & -1 & 0 & 0 & 0 & 0 & 0 & 5 \\ 0 & 0 & 0 & 0 & 0 & 1 & 1 & 1 & -1 & -1 & 6 \\ 0 & 0 & 0 & 0 & 0 & 1 & 1 & -1 & 0 & 0 & 7 \\ 0 & 0 & 0 & 0 & 0 & 1 & -1 & 0 & 0 & 0 & 8 \\ 0 & 0 & 0 & 0 & 0 & 0 & 0 & 0 & 1 & -1 & 9\end{array}$

$\mathrm{Li}, \mathrm{Ni}, \mathrm{As}, \mathrm{Cd}$ and $\mathrm{Hg}$ were removed, as they had a FOD<50\%. See Figure C.1.

The statistical analysis including all samples was performed with the ilr coordinates found using the ilr binary partition shown above. 


\section{E.2.2 Waste samples}

\section{$\mathrm{Cr}$ Li Al Ti Mn Fe Co Ni Cu As Sb Pb}

$\begin{array}{llllllllllll}1 & 1 & 1 & 1 & 1 & 1 & -1 & -1 & -1 & -1 & -1 & -1\end{array}$

$\begin{array}{llllllllllllll}1 & 1 & 1 & -1 & -1 & -1 & 0 & 0 & 0 & 0 & 0 & 0\end{array}$

$\begin{array}{llllllllllll}1 & 1 & -1 & 0 & 0 & 0 & 0 & 0 & 0 & 0 & 0 & 0\end{array}$

$\begin{array}{ccccccccccc}1-1 & 0 & 0 & 0 & 0 & 0 & 0 & 0 & 0 & 0 & 0\end{array}$

$\begin{array}{llllllllllll}0 & 0 & 0 & 1 & 1 & -1 & 0 & 0 & 0 & 0 & 0 & 0\end{array}$

$\begin{array}{llllllllllll}0 & 0 & 0 & 1 & -1 & 0 & 0 & 0 & 0 & 0 & 0 & 0\end{array}$

$\begin{array}{llllllllllll}0 & 0 & 0 & 0 & 0 & 0 & 1 & 1 & 1 & -1 & -1 & -1\end{array}$

$\begin{array}{lllllllllllll}0 & 0 & 0 & 0 & 0 & 0 & 1 & 1 & -1 & 0 & 0 & 0\end{array}$

$\begin{array}{lllllllllllllllllllllll}0 & 0 & 0 & 0 & 0 & 0 & 1 & -1 & 0 & 0 & 0 & 0\end{array}$

$\begin{array}{lllllllllllll}0 & 0 & 0 & 0 & 0 & 0 & 0 & 0 & 0 & 1 & 1 & -1\end{array}$

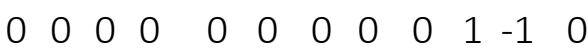

$\mathrm{Zn}, \mathrm{Cd}$ and $\mathrm{Hg}$ were removed, as they had a FOD $<50 \%$. See Figure C.2.

The statistical analysis including waste samples was performed with the ilr coordinates found using the ilr binary partition shown above.

\section{E.3 MANOVA}

\section{E.3.1 All samples}

Two MANOVAS were conducted on all samples, where the grouping of the ilr coordinates was based on:

- Polymer types (PET, PE, PP and PS): $p<2.2 e-16$

- Origin (Sample group 1-4: waste, reprocessed from households, reprocessed from industry, virgin): $p<2.2 e-16$

As $p<0.05$ for both MANOVAS there is a significant difference of metal concentrations between different polymer types and between different groups of origin.

\section{E.3.2 Waste samples}

Three MANOVAS were conducted on the waste samples, where the grouping of the ilr coordinates was based on:

- Washing (non-washed and washed): $p=0.00582$

- Previous use (food contact and non-food contact): $p=0.1846$

- Source (residual and source separated): $P=0.1264$

The MANOVA of the grouping based on previous use only included the part of the waste samples from source separated plastic waste (sample group 1a), as these were the only samples where items of food contact had been separated from those of non-food contact.

The MANOVAs show that there is a significant difference between washed and non-washed waste samples, whereas there is no significant difference when it comes to the source or the previous use. 


\section{E.4 Hotellings $\mathrm{T}^{2}$ test and bootstrap}

\section{E.4.1 Grouping based on polymer (PET, PE, PP, PS)}

The level of significance is adjusted to $\alpha_{1}$ according to Edjabou (2016).

$\alpha_{1}=\frac{\alpha_{0}}{G \cdot \frac{G-1}{2}}$

where $\alpha_{0}=$ the original level of significance of 0.05 and $G$ is the number of groups (here $G=4-P E T, P E$, PP and PS). The adjusted level of significance, $\alpha_{1}$, is therefore 8.3E-3.

The $p$-values for the Hotelling $T$ tests pairwise comparison are presented in Table D.1. The Hotelling T Tests show that there is a significant difference between all types of polymers, except PE and PS.

Table E.1 p-values for all pairwise Hotelling T tests for grouping based on origin of the sample. $p$ values indicating significant difference are highlighted in red.

\begin{tabular}{|l|c|c|c|c|}
\hline$p$-values & PET & PE & PP & PS \\
\hline PET & & 0 & $2.8 \mathrm{E}-12$ & $5.3 \mathrm{E}-8$ \\
\hline PE & 0 & & $2.5 \mathrm{E}-3$ & $1.6 \mathrm{E}-2$ \\
\hline PP & $2.8 \mathrm{E}-12$ & $2.5 \mathrm{E}-3$ & & $1.8 \mathrm{E}-5$ \\
\hline PS & $5.3 \mathrm{E}-8$ & $1.6 \mathrm{E}-2$ & $1.8 \mathrm{E}-5$ & \\
\hline
\end{tabular}

The level of significance was adjusted further for the bootstrap analysis according to Edjabou (2016).

$\alpha_{2}=\frac{\alpha_{1}}{P}$

where $\mathrm{P}$ is the number of dependent variables (here $\mathrm{P}=10-\mathrm{Cr}, \mathrm{Al}, \mathrm{Ti}, \mathrm{Mn}, \mathrm{Fe}, \mathrm{Co}, \mathrm{Cu}, \mathrm{Zn}, \mathrm{Sb}, \mathrm{Pb}$ ). The adjusted level of significance, $\alpha_{1}$, is therefore 8.3E-4. 

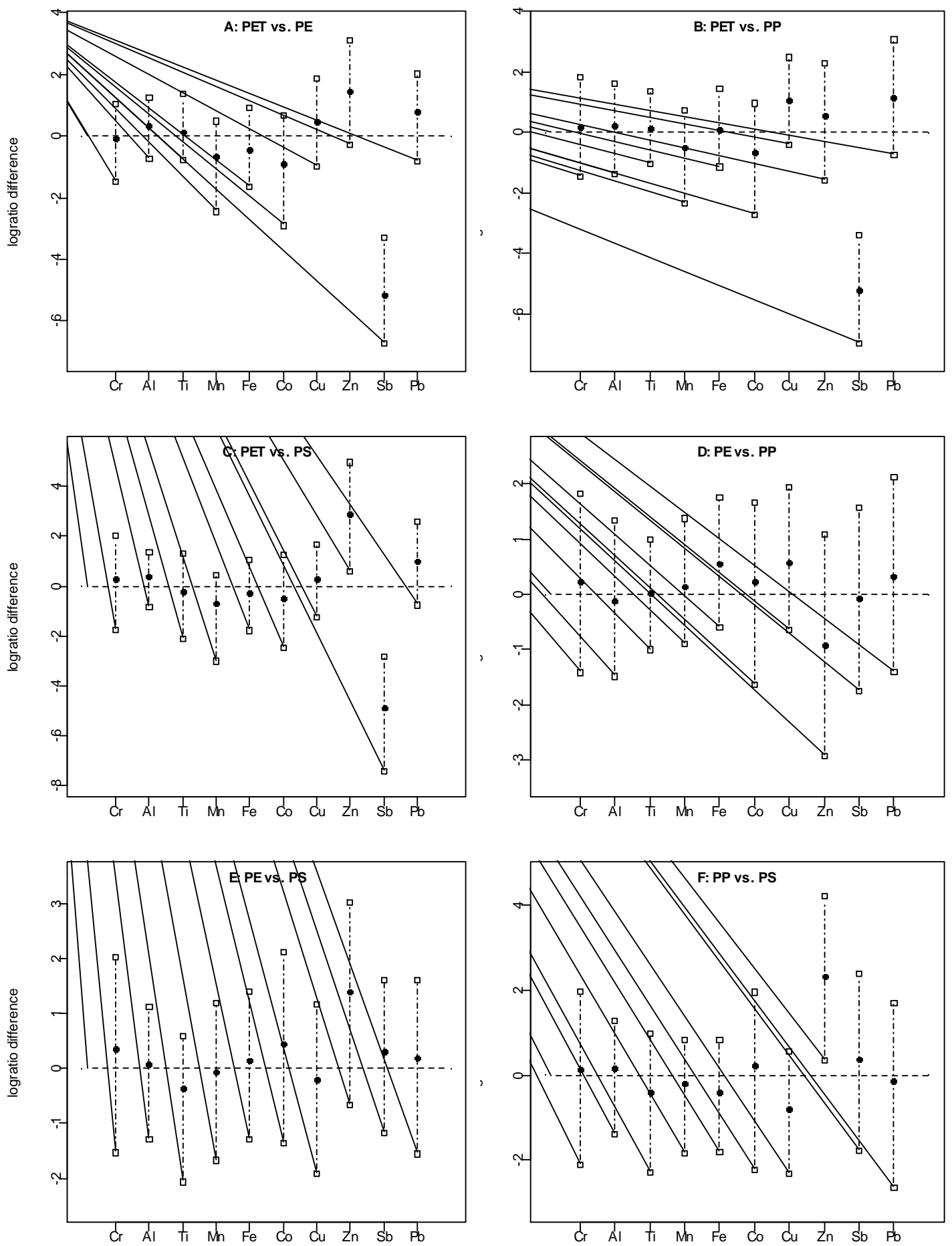

Figure E.1 Pairwise comparison between polymer types of individual metals based on bootstrap percentile confidence intervals for log-ratio difference between: (A) PET and PE, (B) PET and PP, (C) PET and PS, (D) PE and PP, (E) PE and PS, and (F) PP and PS. The filled circles represent the difference between the means and the vertical dashed lines are the percentile intervals. The difference is not significant if a vertical dashed line crosses the 0 -line on the $x$ axis. 


\section{E.4.2 Grouping based on origin (waste, rHHW, rIW and virgin)}

The level of significance is adjusted to $\alpha_{1}$ according to Edjabou (2016).

$\alpha_{1}=\frac{\alpha_{0}}{G \cdot \frac{G-1}{2}}$

where $\alpha_{0}=$ the original level of significance of 0.05 and $G$ is the number of groups (here $G=4$ - waste, $\mathrm{rIW}, \mathrm{rHHW}$ and virgin). The adjusted level of significance, $\alpha_{1}$, is therefore 8.3E-3.

The p-values for all the pairwise Hotteling T tests are given in Table D.2 and shows that the difference between all groups are significant, since all $p$ values $<\alpha_{1}$

Table E.2 p-values for all pairwise Hotelling T tests for grouping based on origin of the sample. pvalues indicating significant difference are highlighted in red.

\begin{tabular}{|l|c|c|c|c|}
\hline $\mathbf{p}$-values & Virgin & Reprocessed from IW & Reprocessed from HHW & Waste \\
\hline Virgin & & $6.4 \mathrm{E}-4$ & $8.5 \mathrm{E}-5$ & 0 \\
\hline Recycled from IW & $6.4 \mathrm{E}-4$ & & $1.4 \mathrm{E}-4$ & $1.9 \mathrm{E}-7$ \\
\hline Recycled from HHW & $8.5 \mathrm{E}-5$ & $1.4 \mathrm{E}-4$ & & $4.5 \mathrm{E}-7$ \\
\hline Waste & 0 & $1.9 \mathrm{E}-7$ & $4.5 \mathrm{E}-7$ & \\
\hline
\end{tabular}

The level of significance was adjusted further for the bootstrap analysis according to Edjabou (2016).

$\alpha_{2}=\frac{\alpha_{1}}{P}$

where $\mathrm{P}$ is the number of dependent variables (here $\mathrm{P}=10-\mathrm{Cr}, \mathrm{Al}, \mathrm{Ti}, \mathrm{Mn}, \mathrm{Fe}, \mathrm{Co}, \mathrm{Cu}, \mathrm{Zn}, \mathrm{Sb}, \mathrm{Pb}$ ). The adjusted level of significance, $\alpha_{1}$, is therefore 8.3E-4. 

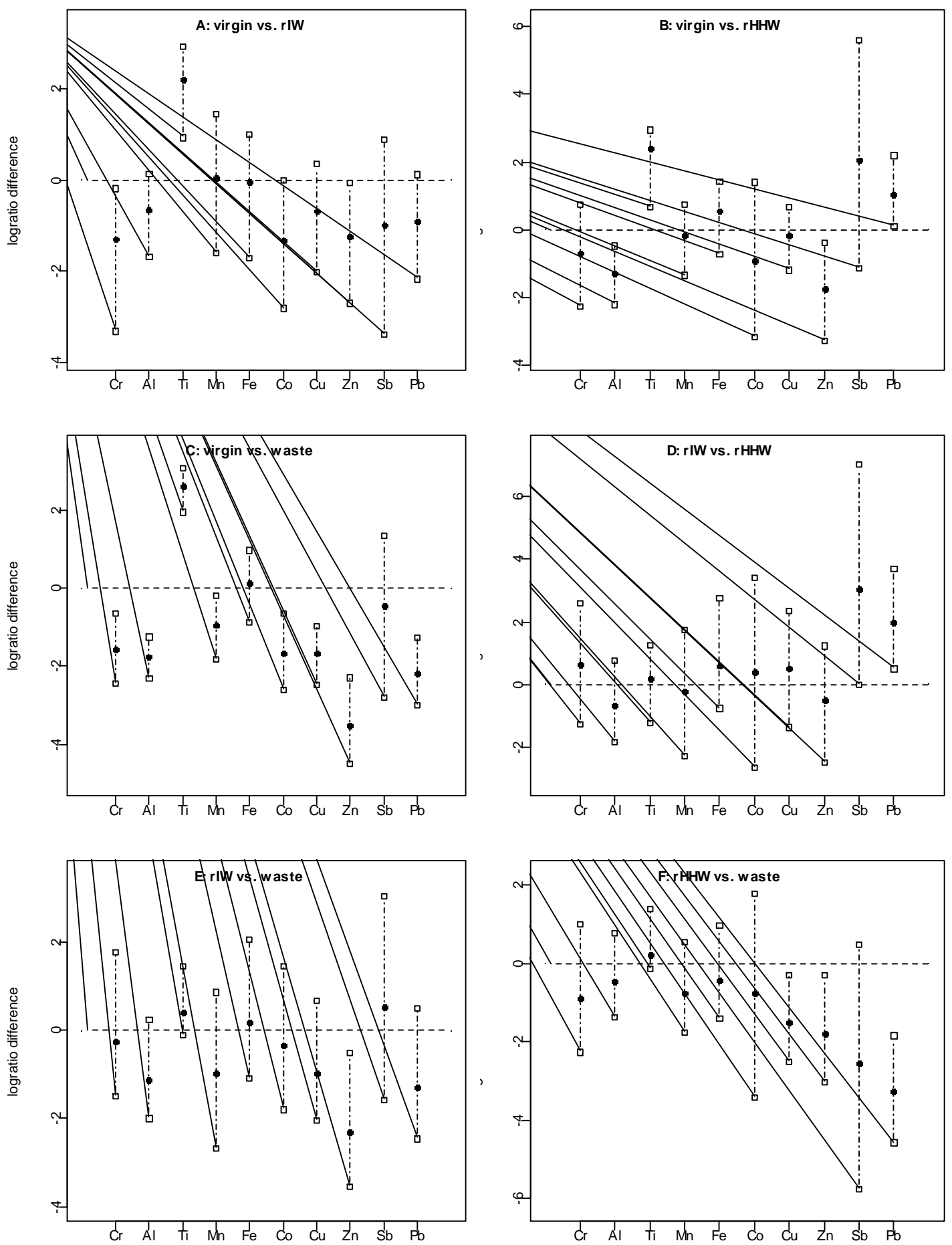

Figure E.2 Pairwise comparison between polymer types of individual metals based on bootstrap percentile confidence intervals for log-ratio difference between: (A) virgin and reprocessed waste from industry (rIW), (B) virgin and reprocessed waste from households ( $\mathrm{rHHW}$ ), (C) virgin and waste, (D) rIW and rHHW, (E) rIW and waste, and (F) rHHW and waste. The filled circles represent the difference between the means and the vertical dashed lines are the percentile intervals. The difference is not significant if a vertical dashed line crosses the 0 -line on the $x$-axis. 


\section{E.4.3 Grouping based on washing - waste samples only}

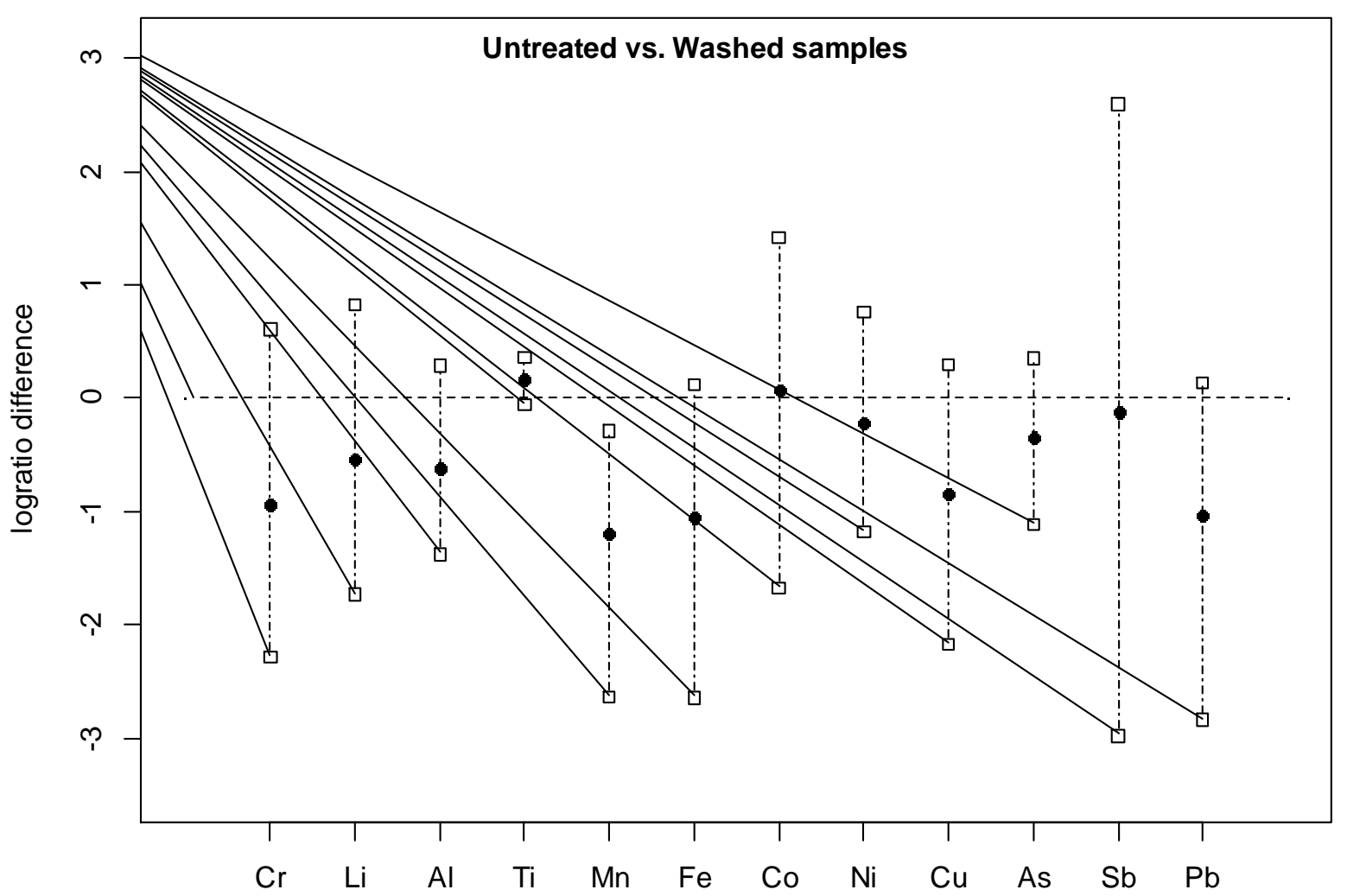

Figure E.3 Pairwise comparison between polymer types of individual metals based on bootstrap percentile confidence intervals for log-ratio difference between untreated and washed plastic waste samples. The filled circles represent the difference between the means and the vertical dashed lines are the percentile intervals. The difference is not significant if a vertical dashed line crosses the 0 -line on the $\mathrm{x}$-axis. 


\section{Appendix F References}

EC (European Commission), 2008. Regulation (EC) No 1272/2008 of the European Parliament and of the council on classification, labelling and packaging of substances and mixtures, amending and repealing Directives 67/548/EEC and 1999/45/EC, and amending Regulation (EC) No 1907/2006. Official Journal of the European Union. L353, 1-1355.

ECHA (European Chemical Agency), 2017. Titanium dioxide proposed to be classified as suspected of causing cancer when inhaled. https://echa.europa.eu/da/-/titanium-dioxideproposed-to-be-classified-as-suspected-of-causing-cancer-when-inhaled [11/01/2018]

Edjabou, M.E., 2016. Composition of municipal solid waste in Denmark. PhD thesis.

Edjabou, M.E., Martín-Fernández, J.A., Scheutz, C., Astrup, T.F., 2017. Statistical analysis of solid waste composition data: Arithmetic mean, standard deviation and correlation coefficients. Waste Management. https://doi.org/10.1016/j.wasman.2017.08.036 Egozcue, J.J., Pawlowsky-Glahn, V., Mateu-Figueras, G., Barceló-Vidal, C., 2003. Isometric Logratio Transformations for Compositional Data Analysis. Mathematical Geology. https://doi.org/10.1023/A:1023818214614

Hahladakis, J.N., Velis, C.A., Weber, R., lacovidou, E., Purnell, P., 2018. An overview of chemical additives present in plastics: Migration, release, fate and environmental impact during their use, disposal and recycling. Journal of Hazardous Materials. https://doi.org/10.1016/j.jhazmat.2017.10.014

Hansen, E., 2013. Hazardous substances in plastic materials. Report by COWI and Danish Technological Institute on behalf of the Norwegian Environment Agency. 